\title{
Unbound or Distant Planetary Mass Population Detected by Gravitational Microlensing
}

T. Sumi ${ }^{1,2,3}$, K. Kamiya ${ }^{1,3}$, A. Udalski ${ }^{4,5}$, D.P. Bennettet, , I.A. Bond ${ }^{1,7}$, F. Abe ${ }^{1,3}$, C.S. Botzler $^{1,8}$, A. Fukui ${ }^{1,3}$, K. Furusawa ${ }^{1,3}$, J.B. Hearnshaw ${ }^{1,9}$, Y. Itow ${ }^{1,3}$, P.M. Kilmartin ${ }^{1,10}$, A. Korpela ${ }^{1,11}$, W. Lin ${ }^{1,7}$, C.H. Ling ${ }^{1,7}$, K. Masuda ${ }^{1,3}$, Y. Matsubara ${ }^{1,3}$, N. Miyake ${ }^{1,3}$, M. Motomura $^{1,3}$, Y. Muraki ${ }^{1,12}$, M. Nagaya ${ }^{1,3}$, S. Nakamura ${ }^{1,3}$, K. Ohnishi ${ }^{1,13}$, T. Okumura ${ }^{1,3}$, Y.C. Perrott ${ }^{1,14}$, N. Rattenbury ${ }^{1,8}$, To. Saito ${ }^{1,15}$, T. Sako ${ }^{1,3}$, D.J. Sullivan ${ }^{1,11}$, W.L. Sweatman ${ }^{1,7}$, P.J. Tristram ${ }^{1,8}$, P.C.M. Yock ${ }^{1,8}$, M.K. Szymański ${ }^{4,5}$, M. Kubiak ${ }^{4,5}$, G. Pietrzyński ${ }^{4,5,16}$, R. Poleski $^{4,5}$, I. Soszyński ${ }^{4,5}$, Ł. Wyrzykowski ${ }^{4,17}$, K. Ulaczyk ${ }^{4,5}$

${ }^{1}$ Microlensing Observations in Astrophysics (MOA) Collaboration.

2 Department of Earth and Space Science, Osaka University, Osaka 560-0043, Japan.

${ }^{3}$ Solar-Terrestrial Environment Laboratory, Nagoya University, Nagoya 464-8601, Japan.

${ }^{4}$ Optical Gravitational Lens Experiment (OGLE) Collaboration.

${ }^{5}$ Warsaw University Observatory, Al. Ujazdowskie 4, 00-478 Warszawa, Poland.

${ }^{6}$ Department of Physics, University of Notre Dame, Notre Dame, IN 46556, USA.

${ }^{7}$ Institute of Information and Mathematical Sciences, Massey University, Private Bag 102904, North Shore Mail Centre, Auckland 0745, New Zealand.

${ }^{8}$ Department of Physics, University of Auckland, Private Bag 92019, Auckland 1142, New Zealand.

${ }^{9}$ Dept. of Physics and Astronomy, University of Canterbury, Christchurch 8140, NewZealand. ${ }^{10}$ Mt. John University Observatory, University of Canterbury, P.O. Box 56, Lake Tekapo 8770, New Zealand.

${ }^{11}$ School of Chemical and Physical Sciences, Victoria University, Wellington 6140, New Zealand.

12 Department of Physics, Konan University, Nishiokamoto 8-9-1, Kobe 658-8501, Japan.

${ }_{13}$ Nagano National College of Technology, Nagano 381-8550, Japan.

${ }^{14}$ Cavendish Laboratory, Cambridge University, J. J. Thomson Avenue, CB3 OHE Cambridge, UK.

15 Tokyo Metropolitan College of Industrial Technology, Tokyo 116-8523, Japan.

16 Universidad de Concepción, Departamento de Astronomia, Casilla 160-C, Concepción, Chile.

${ }^{17}$ Institute of Astronomy, Cambridge University, Madingley Rd., CB3 0HA Cambridge, UK.

Since 1995, more than 500 exoplanets have been detected using different techniques $\stackrel{1}{\underline{1}} \underline{2}$, of which 11 were detected with gravitational microlensing $\underline{\underline{3}}, \underline{\underline{4}}$. Most of these are gravitationally bound to their host stars. There is some evidence of free-floating planetary mass objects in young star-forming regions ${ }^{5,}, \underline{6}, \underline{7}, \underline{8}$, but 
these objects are limited to massive objects of 3 to 15 Jupiter masses with large uncertainties in photometric mass estimates and their abundance. Here, we report the discovery of a population of unbound or distant Jupiter-mass objects, which are almost twice $\left(1.8_{-0.8}^{+1.7}\right)$ as common as main-sequence stars, based on two years of gravitational microlensing survey observations toward the Galactic Bulge. These planetary-mass objects have no host stars that can be detected within about ten astronomical units by gravitational microlensing. However a comparison with constraints from direct imaging ${ }^{9}$ suggests that most of these planetary-mass objects are not bound to any host star. An abrupt change in the mass function at about a Jupiter mass favours the idea that their formation process is different from that of stars and brown dwarfs. They may have formed in proto-planetary disks and subsequently scattered into unbound or very distant orbits.

In a gravitational microlensing event, a foreground lens object is detected as a result of the characteristic magnification of a background source star as it passes behind the gravitational field of the lens $\frac{10}{}$. The lens object is detected by means of its mass and not its luminosity. The duration of the magnification is parameterized by the Einstein radius cross-

ing time, $t_{\mathrm{E}} \sim \sqrt{M / M_{\mathrm{J}}}$ days, where $M_{\mathrm{J}}=9.5 \times 10^{-4} M_{\odot}$ is Jupiters mass. Thus, microlensing can detect faint planetary mass objects - which are either unbound to any host star $\underline{11,12}$ or are in very wide orbits $\underline{\underline{13}}$ - as short-timescale events with $t_{\mathrm{E}}<2$ days. Although $t_{\mathrm{E}}$ also depends on the distance and transverse velocity of the lens (see Supplementary Information), the observed $t_{\mathrm{E}}$ distribution can be used as a statistical probe of the mass function of the lens objects because the spatial and velocity distributions in the Galactic disk and bulge are reasonably well known.

The Microlensing Observations in Astrophysics (MOA) $\underline{\underline{14}}$ and Optical Gravitational Lensing Experiment (OGLE) $\frac{15}{\underline{ }}$ groups both conduct microlensing surveys toward the Galactic bulge. The second phase of MOA, called MOA-II, carries out a high cadence photometric survey of 50 million stars in bulge fields with a cadence of 10-50 minutes. This strategy enables MOA to detect very short events with $t_{\mathrm{E}}<2$ days, which were quite rare in previous microlensing surveys that had lower cadences $\frac{12}{2}, 14,16,17$.

In this analysis of the 2006-2007 MOA-II data set, light curves of genuine microlensing events were distinguished from intrinsic variables and artefacts by several empirical criteria, which have been developed in previous microlensing surveys $\underline{\underline{14}}, \underline{15}, \underline{16}, 17,18, \underline{19}$. The light curves must have a single brightening episode of more than three consecutive significant data points with a constant baseline, and should be well fitted by a theoretical microlensing model $\underline{10}$ with a well constrained $t_{\mathrm{E}}$ (see the Supplementary Information).

Although there are a thousand microlensing events in this sample, only 474 well characterized events have passed our strict selection criteria. Ten of these events have $t_{\mathrm{E}}<2$ days (see Fig. 1 and Table 11) thus indicating planetary-mass lenses. We have confirmed that this event sample has no significant contamination by possible background effects includ- 
ing: (1) cosmic-ray hits, (2) fast-moving objects, (3) cataclysmic variables (4) background supernovae, (5) binary microlensing events, and (6) microlensing by high-velocity stars and Galactic halo stellar remnants. For example, effect (1) is excluded because cosmic-rays never hit the same place in four consecutive images, microlensing model fits for effects (2) to (5) produce a high $\chi^{2}$ and unphysical values of parameters, and effect (6) is excluded by propermotion and radial-velocity observations (see Supplementary Information). After the MOA event selection was complete, the MOA group requested additional independent light-curve data of these short events from the OGLE group. Seven of the ten events with $t_{\mathrm{E}}<2$ days were also observed by OGLE-III $\underline{15}$, and none of them have any other brightening in the eightyear OGLE-III light curves. For six of these seven events, there are OGLE data obtained during the lensing event that confirm the predictions of the MOA microlensing models. Thus, the OGLE data confirms the microlensing interpretation of these short events.

The detection efficiencies for this analysis were estimated with a Monte Carlo simulation $\underline{14}, 17$. We simulated 20 million artificial events to evaluate the detection efficiency as a function of $t_{\mathrm{E}}$, yielding $\epsilon\left(t_{\mathrm{E}}\right) \sim 1 \%, 3 \%, 5 \%, 10 \%, 15 \%$ and $10 \%$ at $t_{\mathrm{E}}=0.3,1,2,10,30$ and 100 days, respectively. The details of the efficiency calculations and consistency tests of the selected event distribution are discussed in the Supplementary Information.

The observed $t_{\mathrm{E}}$ distribution is compared to two mass function models in Fig. 2. A model $t_{\mathrm{E}}$ distribution, $\Phi\left(t_{\mathrm{E}}\right)$, can be calculated for an assumed mass function with a standard Galactic mass density and velocity model $14,17,20$. We consider two mass functions. The first is a broken power-law $\underline{20} \underline{21} \mathrm{~d} N / \mathrm{d} M=M^{-\alpha}$, with the power index of $\alpha_{1}=2.0$ for $0.7 \leq M / M_{\odot} \leq 1, \alpha_{2}=1.3$ for $0.08 \leq M / M_{\odot} \leq 0.7$ and $\alpha_{3}$ as a fitting parameter for the brown dwarf regime $0.01 \leq M / M_{\odot} \leq 0.08$. The second is a log-normal function 22 $\mathrm{d} N / \operatorname{dog} M=\exp \left[\left(\log M-\log M_{\mathrm{c}}\right)^{2} /\left(2 \sigma_{\mathrm{c}}^{2}\right)\right]$ with a mean mass $M_{\mathrm{c}}$ and a width in $\log M$ of $\sigma_{\mathrm{c}}$, for $0 \leq M / M_{\odot} \leq 1.0$. For both mass functions, we assume that stars that were initially above $1 M_{\odot}$ have evolved into stellar remnants - white dwarfs, neutron stars or black holes, depending on their initial masses $\underline{\underline{23}}$ (see Fig. 2 and Supplementary Table 3).

The mass functions were constrained by a likelihood analysis, with the likelihood function given by the product of the model probability $\Phi\left(t_{\mathrm{E}}\right)$ of finding $N_{\mathrm{obs}}=474$ events with each of the observed $t_{\mathrm{E}, i}$, that is; $L=\Pi_{i}^{N_{\text {obs }}} \Phi\left(t_{\mathrm{E}, i}\right) \epsilon\left(t_{\mathrm{E}, i}\right)$.

We have evaluated the likelihood distributions for these mass functions both with and without the $t_{\mathrm{E}}<2$ events, but the inclusion of the events with $t_{\mathrm{E}}<2$ days makes little difference. The results are shown in Supplementary Table 3 and Supplementary Figs 6 and 7. Fig. 2 indicates that both models match the data well for $t_{\mathrm{E}} \geq 2$ days, but at $t_{\mathrm{E}}<2$ days, the ten observed events are well above the model predictions. The power-law and log-normal models predict 1.5 and 2.5 events with $t_{\mathrm{E}}<2$ days, respectively, and the corresponding Poisson probabilities for the ten observed events are $4 \times 10^{-6}$ and $3 \times 10^{-4}$. Thus, we feel confident in adding a new planetary-mass population.

For simplicity, we chose a planetary-mass function model with a $\delta$-function in mass $M_{\mathrm{PL}}$ and a fraction of all objects in the planetary-mass populations $\Phi_{\mathrm{PL}}$. The values of $\left(M_{\mathrm{PL}} / M_{\odot}, \Phi_{\mathrm{PL}}\right)$ derived from the likelihood analysis are $\left(1.1_{-0.6}^{+1.2} \times 10^{-3}, 0.49_{-0.13}^{+0.13}\right)$ and $\left(0.83_{-0.51}^{+0.96} \times 10^{-3}, 0.46_{-0.15}^{+0.17}\right)$ for the power-law and log-normal models, respectively. The 
contours are shown in Fig. 3, Both models for $\Phi\left(t_{\mathrm{E}}\right)$ provide good fits to the entire $t_{\mathrm{E}}$ distribution, as shown in Fig. 2. The power-law and log-normal models imply $1.9_{-0.8}^{+1.3}$ and $1.8_{-0.8}^{+1.7}$ times as many unbound or distant Jupiter-mass objects as main-sequence stars in the mass range $0.08<M / M_{\odot}<1.0$, respectively. These planetary-mass objects are at least 1.5 times as frequent as planets with host stars (see Supplementary Information). We tested a third mass function that has fewer massive stars and brown dwarfs 24 , and found that the resultant planetary-mass function parameters are consistent with above values (see the Supplementary Information).

The lenses for these short events could be either free-floating planets or planets with wide separations of more than about ten astronomical units (AU) from their host stars, for which we cannot detect the host star in the light curves $\frac{25}{}$. However, direct imaging, with adaptive optics, of planets orbiting young stars places upper limits on planets at wide separations. The Gemini Planet Imager has set upper limits $\underline{\underline{9}}$ on the number of stars with Jupiter-mass planets at semi-major axes of 10-500 AU. From these results, we estimate that $<0.4$ of the 1.8 planetary-mass objects per star are likely to be bound to stars at orbital separations of $<500 \mathrm{AU}$ (see Supplementary Information Section 8). Hence, more than $75 \%$ of these planetary mass objects are probably unbound to stars if their typical mass is a Jupiter-mass or more.

Since the $\delta$-function planetary models are not likely to be realistic, we also tested a fourth mass function that is identical to the first, broken-power-law model except for having a power-law form in the planetary mass regime, $M<0.01 M_{\odot}$. This yields a planetary-mass index of $\alpha_{\mathrm{PL}}=1.3_{-0.4}^{+0.3}$, which is much steeper than the brown dwarf slope of $\alpha_{3}=0.49_{-0.27}^{+0.24}$, indicating that they are distinct populations (see Supplementary Information).

Planet-formation theories predict that dynamical instabilities in planetary systems with multiple giant planets could scatter many of these planets into unbound orbits $\frac{26}{}$, as well as some into large separations ${ }^{27}$. Recent observations also indicate that planet-planet scattering plays an important part in moving giant planets into short-period orbits $\underline{28}, 2 \underline{29}$. The planetary-mass population that we have identified here may have formed in protoplanetary disks at much smaller separations and then been scattered into unbound or very distant orbits.

Supplementary Information accompanies the paper on www.nature.com/nature.

Acknowledgements MOA thanks the support by the JSPS and MEXT of Japan, and the Marsden Fund of New Zealand. D.P.B. acknowledge supports by the NSF and NASA. The OGLE project is grateful for funding from the European Research Council Advanced Grants Program.

Author contributions T.S. and K.K. conducted data reduction and statistical analysis. A.U. produced OGLE-III light curves. I.A.B. generated the extended MOA-II light curves. 
D.P.B. conducted the detail analysis of binary events. T.S. wrote the manuscript. D.P.B. and I. A. B. edited the manuscript. All authors contributed to the observation and maintenance of observational facilities, discussed the results and commented on the manuscript.

Author Information Reprint and permissions information is available at npg.nature.com/reprintsandpermissions. The authors declare no competing financial interests. Correspondence and requests for materials should be addressed to T.S. (e-mail: sumi@ess.sci.osaka-u.ac.jp). 

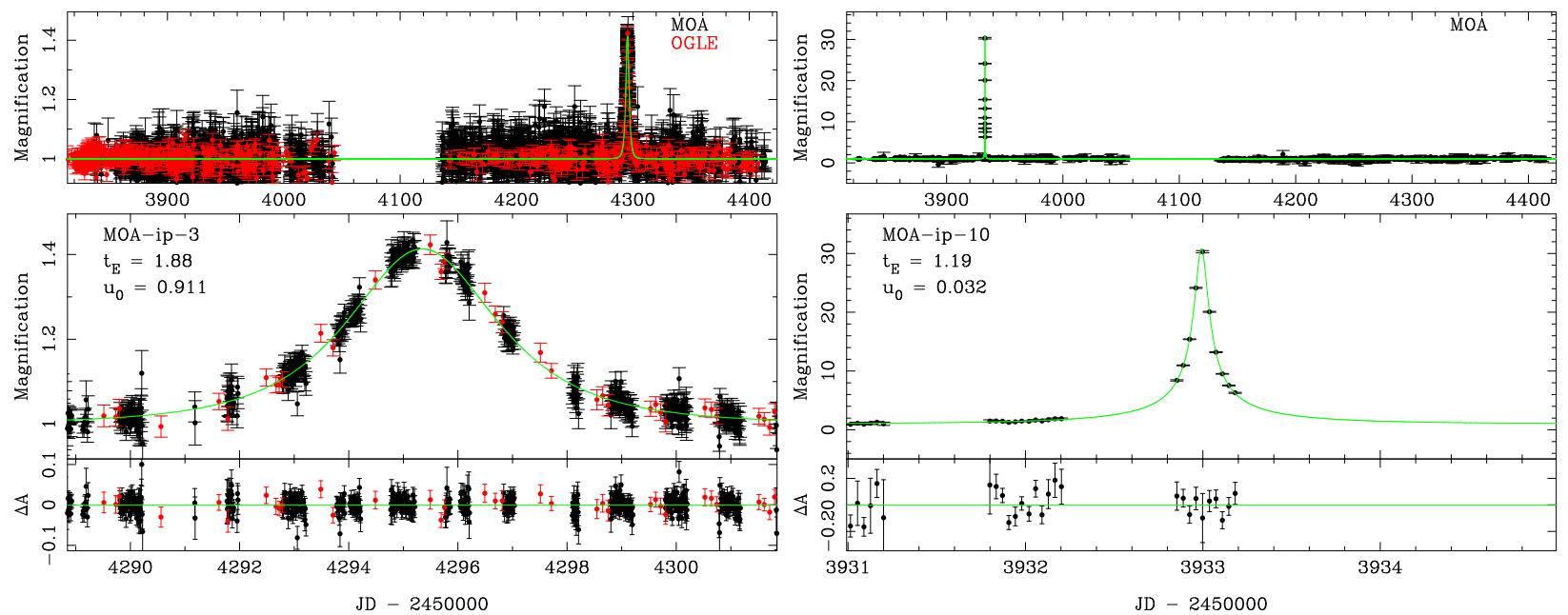

Figure 1. - Light curves of event MOA-ip-3 and MOA-ip-10. These have the highest signal to noise ratio amongst the ten microlensing events with $t_{\mathrm{E}}<2$ days (see Supplementary Fig. 1 for the others). MOA data are in black and OGLE data are in red with error bars indicating the s.e.m. The green lines represent the best-fit microlensing model light curves. For each event, the upper panel shows the full two-year light curve, the middle panel is a close-up of the light curve peak, and the bottom panel shows the residuals from the best-fit model in units of magnification, $\Delta A$. $u_{0}$ indicates the source-lens impact parameter in unit of the Einstein radius. The second phase of MOA, MOA-II, carried out a very high cadence photometric survey of 50 million stars in 22 bulge fields (of $2.2 \mathrm{deg}^{2}$ each) with a $1.8 \mathrm{~m}$ telescope at Mt. John Observatory in New Zealand. MOA detects 500-600 microlensing events with 8 months observation every year. In 2006-2007, MOA observed two central bulge fields every 10 minutes, and other bulge fields with a 50 minutes cadence, which result $\sim 8250$ and 1660-2980 images, respectively. This strategy enabled MOA to detect very short events with $t_{\mathrm{E}}<2$ days. Since 2002, the OGLE-III survey has monitored the bulge with the 1.3-m Warsaw telescope at Las Campanas Observatory, Chile, with a smaller field-ofview but better astronomical seeing than MOA. The OGLE-III observing cadence was 1-2 observations per night, but the OGLE photometry is usually more precise and fills gaps in the MOA light curves due to the difference in longitude. 


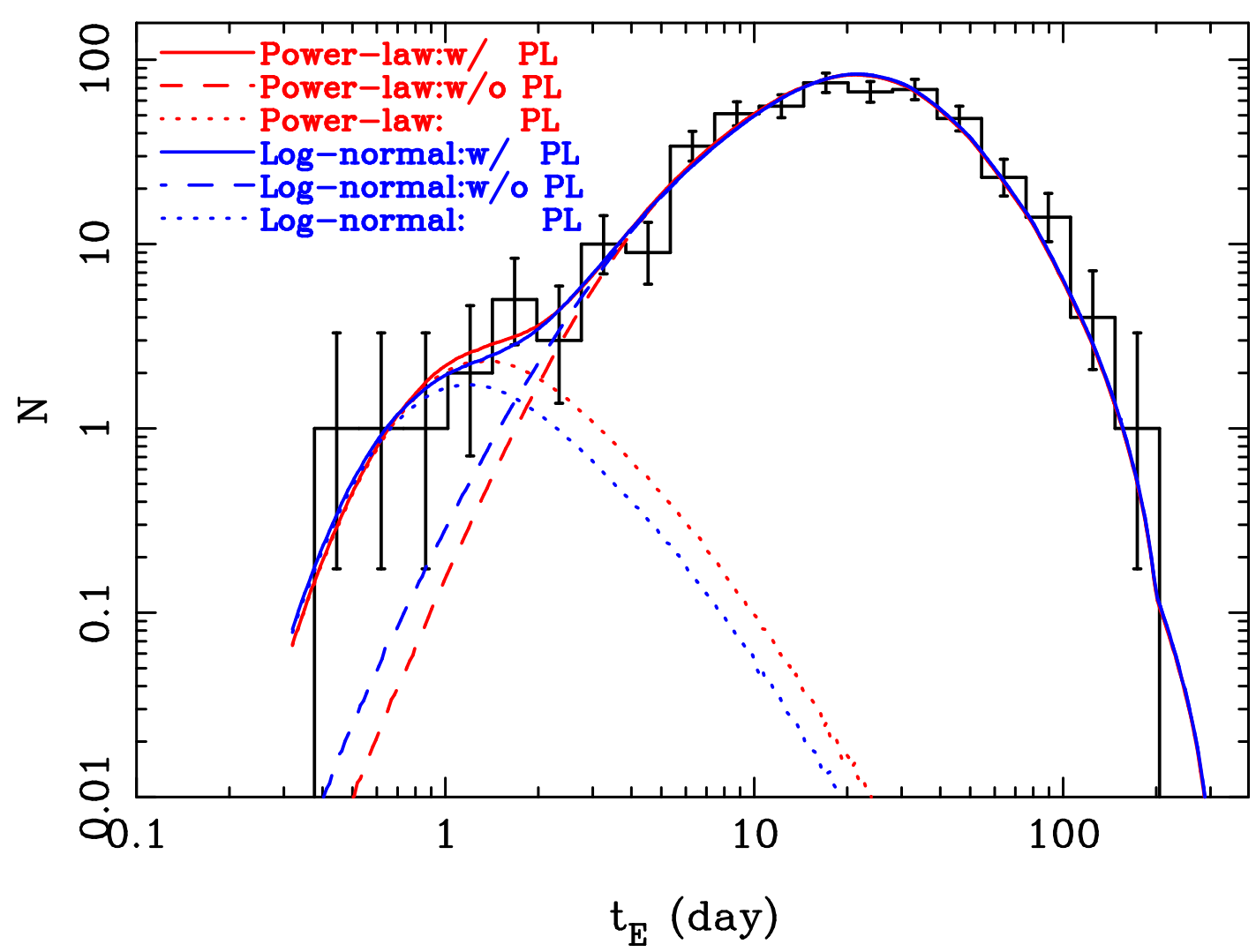

Figure 2.- Observed and theoretical distributions of the event timescale, $t_{\mathrm{E}}$. The black histogram represents the number $N$ of observed 474 microlensing events in each bin with error bars indicating the s.e.m. The red and blue lines indicate the best-fit models with (1) the power-law and (2) log-normal mass functions, respectively. For both mass functions, we assume that stars that were initially above $1 M_{\odot}$ have evolved into stellar remnants white dwarfs, neutron stars or black holes depending on the initial mass. The number of remnants is determined by extending the upper main sequence power-law $\alpha_{1}=2.0$ to 100 $M_{\odot}$, and the final remnant mass distributions are given by Gaussians $\underline{23}$ (see Supplementary Table 3). Each model is multiplied by the detection efficiencies. In each model, dashed lines indicate models for stellar, stellar remnant and brown dwarf populations, and the dotted lines represent the planetary-mass population (PL). Solid lines are the sums of these populations, and both models fit the data well. 


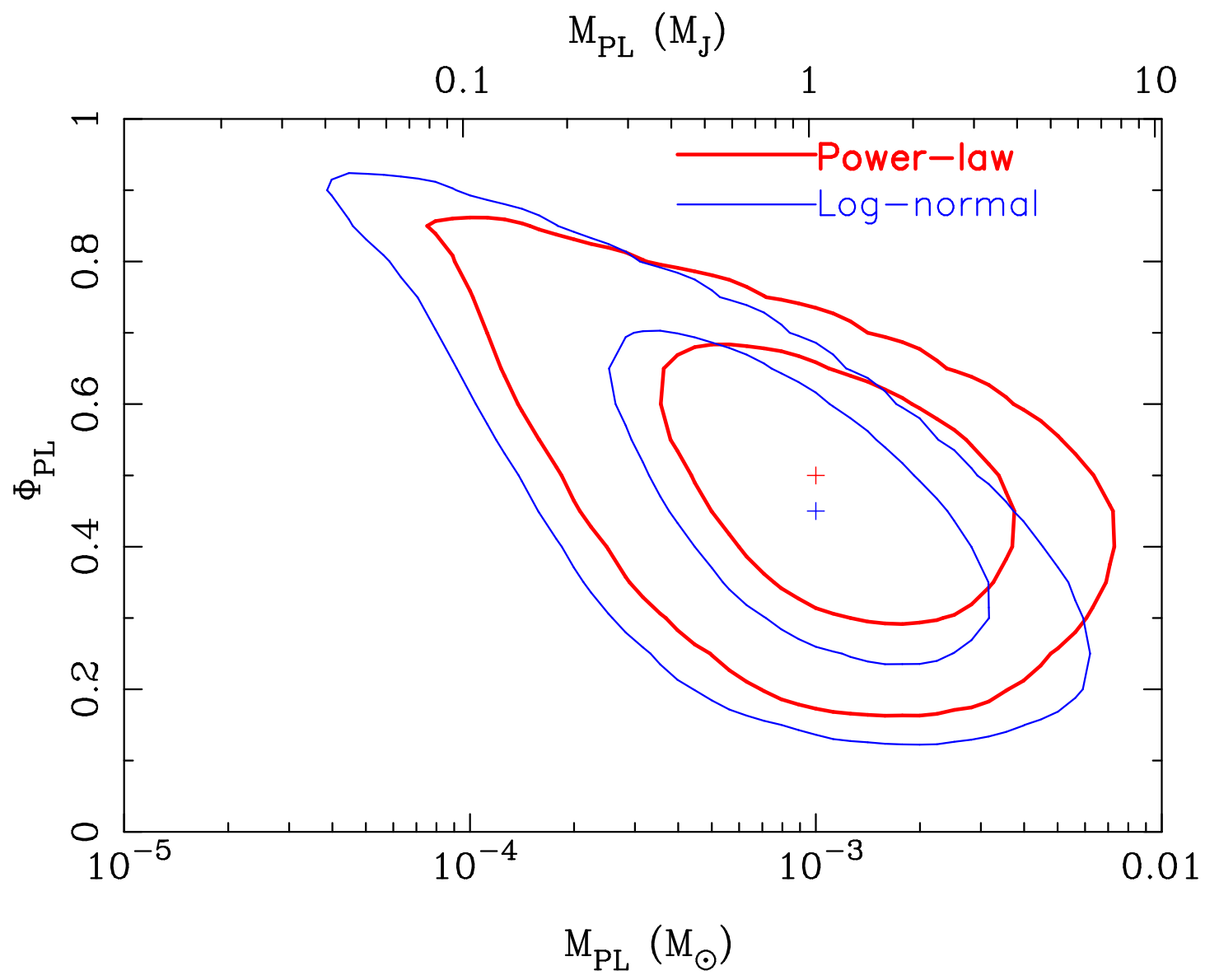

Figure 3.- Likelihood contours for the planetary-mass function parameters. $\Phi_{\mathrm{PL}}$ indicates the fraction of all objects in the planetary-mass population, not including the brown dwarfs that have planetary mass in the tail of the log-normal mass function. $M_{\mathrm{PL}}$ represents their mass. The two sets of contours indicate the $68 \%$ and $95 \%$ confidence levels. The red and blue curves indicate the power-law and log-normal mass functions, respectively, and " +" indicates the maximum likelihood points. The top axis scale is Jupiter masses, $M J=9.5 \times 10^{-4} M_{\odot}$, while bottom axis scale is in Solar masses, $M_{\odot}$. For the power-law model, the likelihoods are evaluated in the $\left(\alpha_{3}, M_{\mathrm{PL}}, \Phi_{\mathrm{PL}}\right)$ space and projected into the $\left(M_{\mathrm{PL}}, \Phi_{\mathrm{PL}}\right)$ plane. The $M_{\mathrm{c}}=0.12$ and $\sigma_{c}=0.76$ parameters are fixed for the log-normal model. The median and $68 \%$ confidence intervals of $\left(M_{\mathrm{PL}} / M_{\odot}, \Phi_{\mathrm{PL}}\right)$ are $\left(1.1_{-0.6}^{+1.2} \times 10^{-3}, 0.49_{-0.13}^{+0.13}\right)$ and $\left(0.83_{-0.51}^{+0.96} \times 10^{-3}\right.$, $\left.0.46_{-0.15}^{+0.17}\right)$ for the power-law and log-normal models, respectively. The results for two models are consistent with each other. The power-law and log-normal models imply $1.9_{-0.8}^{+1.3}$ and $1.8_{-0.8}^{+1.7}$ times as many unbound or distant Jupiter-mass objects as the main sequence stars. $\alpha_{3}$ is consistent with the values derived without planetary population indicating that brown dwarfs are $0.7 \pm 0.3$ times as common as main sequence stars. The numerical values of the models are summarized in supplementary Table 3. 
Table 1. Microlensing Parameters of Short-Timescale Events.

\begin{tabular}{|c|c|c|c|c|c|c|c|c|c|c|}
\hline ID & field & $\begin{array}{l}\text { R.A. } \\
(2000)\end{array}$ & $\begin{array}{l}\text { Dec. } \\
(2000)\end{array}$ & $N_{t_{\mathrm{E}}}$ & $\begin{array}{c}t_{0} \\
\left(\mathrm{JD}^{\prime}\right)\end{array}$ & $\begin{array}{c}t_{\mathrm{E}} \\
\text { (days) }\end{array}$ & $\begin{array}{c}u_{0} \\
\left(R_{\mathrm{E}}\right)\end{array}$ & $A_{\max }$ & $\begin{array}{c}I_{\mathrm{s}} \\
(\mathrm{mag})\end{array}$ & $\begin{array}{l}d_{\min } \\
\left(R_{\mathrm{E} *}\right)\end{array}$ \\
\hline MOA-ip-1 & gb1-4 & $17: 46: 24.506$ & $-34: 30: 36.82$ & 9 & 3883.24171 & $0.73 \pm 0.08$ & $0.028 \pm 0.003$ & 35.6 & 19.7 & 7.0 \\
\hline MOA-ip-3 & gb5-7 & $17: 54: 58.325$ & $-29: 38: 20.68$ & 170 & 4295.34720 & $1.88 \pm 0.12$ & $0.911 \pm 0.096$ & 1.4 & 17.2 & 3.6 \\
\hline MOA-ip-4 & gb5-8 & $17: 54: 24.543$ & -29:13:29.39 & 81 & 3961.38803 & $1.48 \pm 0.12$ & $0.271 \pm 0.061$ & 3.8 & 19.2 & 3.1 \\
\hline MOA-ip-5 & gb9-2 & $17: 57: 17.008$ & $-29: 02: 33.59$ & 69 & 4169.60907 & $1.62 \pm 0.69$ & $0.126 \pm 0.159$ & 8.0 & 19.2 & 2.4 \\
\hline MOA-ip-7 & gb9-5 & $17: 57: 36.678$ & $-29: 59: 40.52$ & 51 & 4370.69496 & $1.82 \pm 0.87$ & $0.143 \pm 0.125$ & 7.0 & 19.4 & 5.2 \\
\hline MOA-ip-8 & gb9-5 & $17: 59: 34.877$ & $-30: 04: 24.04$ & 47 & 4013.14052 & $1.36 \pm 0.15$ & $0.103 \pm 0.016$ & 9.8 & 18.8 & 4.8 \\
\hline MOA-ip-9 & gb10-5 & $17: 57: 52.952$ & $-28: 16: 56.66$ & 16 & 3910.81772 & $0.96 \pm 0.21$ & $0.163 \pm 0.058$ & 6.2 & 19.5 & 3.4 \\
\hline MOA-ip-10 & gb11-9 & 18:09:00.076 & $-32: 18: 39.91$ & 21 & 3932.99205 & $1.19 \pm 0.04$ & $0.032 \pm 0.001$ & 30.8 & 18.8 & 15.0 \\
\hline
\end{tabular}

Note. - $N_{t_{\mathrm{E}}}$ indicates the number of data points within $t_{0} \pm t_{\mathrm{E}}$ and $t_{0}, t_{\mathrm{E}}, A_{\mathrm{max}}$ and $I_{\mathrm{s}}$ indicate the time of peak magnification, the Einstein radius crossing time, the maximum magnification, and the source star magnitude of the best fit models of the MOA data, respectively. JD'=JD-2450000. $u_{0}$ and $d_{\text {min }}$ indicate the sourcelens impact parameter and minimum host star separation in units of the Einstein radii of the planetary mass lens, $R_{\mathrm{E}}$, and possible host star, $R_{\mathrm{E} *}$, respectively. The errors in $t_{\mathrm{E}}$ and $u_{0}$ represent $1-\sigma$ limits. $d_{\min }$ indicates the 2- $\sigma$ limits. MOA-ip-2, MOA-ip-3 and MOA-ip-10 were alerted as MOA-2007-BLG-144, MOA-2007-BLG-309 and MOA-2006-BLG-098 by the MOA real-time alert system (http://www.massey.ac.nz/ iabond/moa). 


\section{Supplementary Information}

This supplement to "Unbound or Distant Planetary Mass Population Detected by Gravitational Microlensing," by Sumi et al. provides additional details of the analysis presented in this paper. The basics of gravitational microlensing is briefly introduced in Section 1. The detailed data analysis and event selection methods are described in Section 2. In Section 3. we discuss possible backgrounds that could conceivably result in false detections of short timescale microlensing event, and we show that the short timescale event sample does not have any significant contamination by false-positive detections. We discuss the simulations used for the detection efficiency calculations in Section 4 . In Section 5, we show that systematic biases of the parameters produced by our analysis are small, and in Section 6. we present the details of the likelihood analysis that measures the substellar mass function. The likelihood analysis for other mass functions are also given in Section 7 . Finally, in Section

8 we compare the frequency of unbound or distant planetary mass objects that of bound planets.

\section{Basic of the gravitational microlensing}

The gravitational microlensing method allows the detection of extremely faint or completely dark objects when their gravitational fields act as a lens to magnify background source stars $\frac{10}{}$. A gravitational lens is characterized by its Einstein ring radius, which is the radius of the ring image seen when the lens and source are perfectly aligned. In a microlensing event, the image separation is too small to resolve, and the observable feature is the changing total magnification due to the lens-source relative motion. The key parameter of a microlensing light curve is the Einstein radius crossing time given by

$$
t_{\mathrm{E}}=\frac{R_{\mathrm{E}}\left(M, D_{\mathrm{l}}, D_{\mathrm{s}}\right)}{v_{\mathrm{t}}}, \text { where } R_{\mathrm{E}}=\sqrt{\frac{4 G M}{c^{2}} \frac{D_{\mathrm{l}}\left(D_{\mathrm{s}}-D_{\mathrm{l}}\right)}{D_{\mathrm{s}}}},
$$

where $v_{\mathrm{t}}$ is the relative transverse velocity between the lens and source, $R_{\mathrm{E}}$ is the Einstein radius, $M$ is the lens mass, $D_{\mathrm{l}}$ and $D_{\mathrm{s}}$ are the distance to the lens and the source from the observer, $G$ is the gravitational constant and $c$ is the speed of light. Although $t_{\mathrm{E}}$ depends on the velocity and distance of the lens object, in addition to its mass, the $t_{\mathrm{E}}$ distribution can be used to probe the mass function of the lens objects statistically. Thousands of microlensing events due to stars in the Galactic disk and bulge have been detected and used to study the stellar mass function and galactic structure $\underline{14}, \underline{16}, \underline{17}, \underline{18}$. 


\section{Data Analysis and Event Selection}

The 2006-2007 MOA-II galactic bulge data consists of $~ 8250$ images of each of the two most densely sampled fields (fields gb5 and gb9) and 1660-2980 images of each of the 20 other less densely sampled fields as shown in Table 1. We observed these galactic bulge fields for about 8 months of each year from the end of February to the beginning of November. This observing strategy is designed to detect single lens events and short timescale anomalies in the light curves of stellar microlensing events due to planets orbiting the lens stars $\mathbf{4}^{4}, 30,31$. The MOA images were reduced with MOA's implementation ${ }^{32}$ of the difference image analysis (DIA) method $33,34,35$. In the DIA method, a high quality, good seeing, reference image is subtracted from each observed image after transforming the reference image to give it the same seeing and photometric scaling as the observed image. This method generally provides more precise photometry in the very crowded Galactic bulge fields than straight PSF-fitting routines, such as DOPHOT $\stackrel{36}{2}$. This is, in part, due to the fact that the Galactic bulge fields are so crowded that virtually all the main sequence stars are not individually resolved. As described in Section 4, the identification of a clear red clump giant population in the data is needed to match the observed MOA luminosity function to the much deeper Hubble Space Telescope luminosity function that describes the source stars. For the gb22 field and some fraction of other fields, totaling about $12 \%$ of the area, a clear red clump population could not be identified in the color magnitude diagram, and these regions were excluded from the analysis for this reason. This lack of a clear red clump giant feature in field gb22 is probably because it is relatively far from the center of the Galaxy. It is generally regions of very high interstellar extinction that prevented the identification of the red clump giant color magnitude diagram feature in portions of the other fields.

The images were taken using the custom MOA-Red wide-band filter, which is equivalent with the sum of the standard Kron/Cousins $R$ and $I$-bands. The instrumental magnitudes of the MOA reference images were calibrated to the Kron/Cousins I-band using OGLE-II photometry map of the Galactic bulge ${ }^{37}$. The mean magnitude zero-point were estimated from the $30 \%$ of MOA-II fields which overlap with the OGLE-II map. We applied this mean zero-point to all fields. Here the error of the calibrated magnitude is estimated to be $\sim 0.25$ mag from the standard deviation of zero-points in overlap fields. Although this calibration is approximate, this does not affect following analysis at all because the luminosity functions, which is the only part of our analysis requiring calibrated magnitudes, are calibrated by using red clump giants, as discussed in Section 4.

The problem of microlensing event selection has been addressed in previous microlensing analyses $\underline{14}, 17,18,19,38,39,40$, and we adopt event selection criteria that are similar to those used in a number of these previous papers. We employ three levels of selection criteria, or cuts, to distinguish genuine microlensing events from intrinsic variable stars and astrophysical and non-astrophysical artifacts.

(1) Cut-0: On a subtracted image, variable objects can be seen as positive or negative point spread function (PSF) profiles depending on whether the target star is brighter 
or fainter than in the reference image. We use a custom implementation of the IRAF task DAOFIND ${ }^{41}$ to detect these variable objects (like microlensing events), with the modification that both positive and negative PSF profiles are searched for simultaneously. This algorithm finds difference image peaks with a signal to noise ratio $(\mathrm{S} / \mathrm{N})$ $>5$ and then applies several additional criteria to avoid the detection of spurious variations that are not associated with stellar variability, such as cosmic ray hits, satellite tracks and electrons leaked from the saturated images of bright stars. The positions of detected objects are checked against those obtained in previous reductions of the field. When no object is cross-referenced, the object is classified as new and added to the list of variable object positions. If an object has previously been detected, the number of detections for this object, $N_{\text {detect }}$, is incremented. We require that $N_{\text {detect }}$ to be $>2$. About $9.7 \times 10^{6}$ variable objects were detected at this stage of the analysis, including a number of image artifacts of various types.

(2) Cut-1: Light curves of the candidates passing Cut-0 were then created using PSF fitting photometry on the difference images. Only objects with more than 500 photometric data points (from 1660-8250 images depending on the field) were used for further analysis. The photometric error bars were calibrated with constant stars in each subfield. We place a 120-day moving window on each light curve and define the baseline flux to be the weighted average flux outside of that window. We require the baseline to have more than 10 data points and have $\chi_{\text {out }}^{2} /$ dof $\leq 3$ for a constant flux fit. As other microlensing surveys have found, the error bar estimates from the photometry code provide only an approximate description of the photometric uncertainty for each measurement. The actual uncertainties depend upon the distribution of stars in the immediate vicinity of the target. We therefore define normalized uncertainties $\sigma_{i}^{\prime} \equiv$ $\sigma_{i} \sqrt{\chi_{\text {out }}^{2} / \text { dof }}$, where $\sigma_{i}$ are the error bar of the measured flux $F_{i}$ for the $i$ th measurement. We then search for positive light curve "bumps" inside the 120-day window, with a "bump" defined as a brightening episode with more than 3 consecutive measurements with excess flux $>3 \sigma^{\prime}$ above the baseline flux, $F_{\text {base. }}$. We define a statistic $\chi_{3+}=$ $\Sigma_{i}\left(F_{i}-F_{\text {base }}\right) / \sigma_{i}^{\prime}$ summed over consecutive points with $F_{i}-F_{\text {base }}>3 \sigma_{i}^{\prime}$. We require that a bump should have $\chi_{3+} \geq 80$ to pass this cut.

(3) Cut-2: The candidate events that pass Cut-1 are fit with a 5-parameter microlensing model assuming a point source and a single lens object $\underline{10}$. The 5 parameters are the Einstein radius crossing time, $t_{\mathrm{E}}$, the time of peak magnification, $t_{0}$, the source-lens impact parameter (in units of the Einstein radius), $u_{0}$, and the source and background fluxes, $F_{\mathrm{s}}$ and $F_{\mathrm{b}}$. Candidate events are rejected when the Markov Chain Monte Carlo (MCMC) fitting method does not converge, because such events generally do not have well constrained parameters and may add a systematic bias in the observed $t_{\mathrm{E}}$ distribution (see Section 5 for more details). We place constraints on the fit $\chi^{2}$ for these microlensing fits. We require that $\chi^{2} /$ dof $<2$ for both the full fit and for the regions near the peak with $\left|t-t_{0}\right|<t_{\mathrm{E}}$ and $\left|t-t_{0}\right|<2 t_{\mathrm{E}}$. We require that $t_{0}$ 
be within the observation period, and we exclude very low-magnification events by requiring $u_{0} \leq 1$ with an error bar of $\sigma_{u_{0}}<0.3$. We also limit the best fit Einstein radius crossing times to the range 0.3 days $\leq t_{\mathrm{E}} \leq 200$ days with an error bar of $\leq 50 \%$ and $\leq 12$ days, and we require that the source should have an (approximately calibrated) $I$-band magnitude brighter than 20.0. We require that the best fit source flux not exceed the cataloged flux on of an apparent source on the reference frame by more than a factor of 3 . This allows for some "negative blending", which can occur if a source happens to be located at a local minima in the flux of unresolved stars.

Of course, most of the cuts on the fit parameters exclude legitimate microlensing events, but we use them because they allow us to exclude the background of non-microlensing events with a relatively simple set of cuts. The cuts on $t_{\mathrm{E}}$ are of particular interest because these will effect the shape of the $t_{\mathrm{E}}$ distribution that is one of the major results of this analysis. The $t_{\mathrm{E}}<200$ day cut (and the use of a 120-day window in Cut-0) excludes a large number of long events, which would be very important if we were trying to measure the high-mass end of the present-day mass function. This is not a problem for this analysis, which focuses on the low-mass end of the mass function. The removal of short events with $t_{\mathrm{E}}<0.3$ days probably does not remove any events from this sample, but there are certainly real events of such short timescales. It will be important to remove this cut in future analyses of larger event samples that will measure the shape of the mass function of the unbound or distant planetary mass objects.

Events with systematic residuals from the best fit model are also rejected, and this cut depends on the significance of the microlensing signal. We define the maximum number of consecutive measurements that are scattered from the best fit model with excess flux more than 2- $\sigma$ and $3-\sigma$ by $N_{2 \sigma}$ and $N_{3 \sigma}$, respectively. We require that $\chi_{3+} \geq 70 N_{2 \sigma}-500$, and $\chi_{3+} \geq 45 N_{3 \sigma}$ or $N_{3 \sigma} \leq 2$.

All event selection criteria are summarized in Table 2. We have selected events which can be well fit with a point-source, single-lens model, and we have omitted binary lens events in this analysis by the cuts above, although some previously unknown planetarymass binary lens events have been found in this analysis (Bennett et al. in preparation). Although we have identified more than a thousand microlensing candidates in this data set, only 474 high quality microlensing events have passed our relatively strict criteria. These strict criteria ensure that $t_{\mathrm{E}}$ is well constrained for each event and that there is no significant contamination by mis-classified events, which might bias the $t_{\mathrm{E}}$ distribution. This potential contamination by mis-classified events is discussed in the next Section.

\section{$3 \quad$ Background Events}

There are a variety of effects that can give signals that bear some resemblance to short timescale microlensing events, so it is important to ensure that our sample of events is 
not contaminated by any of these effects. The potential background effects that we have considered are the following:

(1) Cosmic-ray hits: It is rare for cosmic ray hits to give a signal with the same profile as the observed PSF. So, most cosmic rays are rejected due to their lack of a PSF-like shape. There was a previous claim of the detection of free-floating planetary mass objects by microlensing in the globular cluster M22 based on imaging by the Hubble Space Telescope $(\mathrm{HST})^{42}$, but this claim was retracted within a few months $\underline{\underline{43}}$ as the putative microlensing events were artifacts caused by cosmic ray hits very close to the targets stars on two consecutive images. Our data set is not subject to such confusion for a number of reasons. First, unlike the MOA images, HST images are under-sampled, so that cosmic rays are much more difficult to reject based on their PSF shape in the HST images than in the MOA images. Second, the light curves presented in the M22 paper are maximally under-sampled, with only a pair of consecutive (cosmic-ray split) images that were brighter than the baseline brightness. In contrast, we explicitly require at least 4 consecutive observations that are $>3 \sigma^{\prime}$ above baseline and implicitly require much larger number with our cuts on the fit parameter error bars. Finally, the cosmic ray rate is much lower for ground-based images than for HST images. In fact, each of our $10 t_{\mathrm{E}}<2$ day events have at least 10 observations at significant magnification, so there is no chance of contamination by cosmic ray hits.

(2) Fast Moving objects: Our pipeline conducts PSF photometry at the fixed position where the variable object was first detected on a difference image. If an object moves a significant fraction of PSF during several exposures, the photometry code will begin to underestimate its brightness due to the off-center PSF. With this photometry method, the linear movement of such an object can produce a light curve which has some resemblance to a microlensing light curve. Any small Solar System body, such as an asteroid or Kuiper belt object, with a proper motion of $\sim 1$ asec $h^{-1}$, can mimic a short timescale microlensing event. Dust specs on the camera window can also produce similar light curves due to slight changes in the telescope pointing between different images of the same field. While light curves due to moving objects or dust specs can sometimes be reasonably well fit with a microlensing light curve model, they generally give unphysical light curve parameters, such as a large $u_{0}$ value, a small $t_{\mathrm{E}}$ value, and a fit source brightness, which to bright to be compatible with stars seen in the un-subtracted images. The moving objects in this data sets were all rejected by two independent methods. We have identified 3743 moving objects in this data set by direct measurements of the changing image positions on the difference images, and we found that all the light curves of these objects were rejected due to poor microlensing fits or unphysical microlensing model parameters.

(3) Cataclysmic variables $(\mathrm{CVs})$ : The most important background for this analysis is a particular class of $\mathrm{CVs}$ that can have short timescale brightening episodes and repeat rarely enough so that they might have been missed despite the multi-year, high cadence 
monitoring of the MOA-II bulge fields. Our primary means for identifying CVs is through the microlensing light curve fits. Most of the CVs can be identified from their highly asymmetric light curves, with a steep rise and a slow decline. However, for some CVs, this asymmetry is not seen due to gaps in light curve sampling or large photometric error bars. However, even poorly sampled CVs can generally be distinguished from microlensing light curves because a theoretical microlensing profile fit yields unphysical values such as a very large value of $u_{0}$ and a sources with much brighter baseline fluxes than allowed by the reference images

We identified $418 \mathrm{CVs}$ in our sample from a visual inspection of light curves that failed the cuts on the quality of the microlensing fit or the upper limit on the brightness of the source star. The timescales of the brightening of these CVs range from hours to months. An important property of $\mathrm{CVs}$ is that they repeat $\underline{44}$, and we have used this property to find an upper limit on the CV contamination of our sample. Using an expanded data set running through 2010, we found $208 \mathrm{CVs}$ with more than one outburst. The average interval between microlensing events with the same source is about 100 thousand years if the lens objects are not physically related. The rate of repeating microlensing events due to wide binary lens or source systems is $<1$ in a few hundred events, so very few, if any, of these events are likely to be actual microlensing events. Slight contamination of this CV sample with microlensing events is not a problem because these CV samples are only used for determining an upper limit of CVs which could pass our selection criteria.

This upper limit is derived as follows. Because these CVs were identified by their multiple outbursts instead of the light curve shape, they can be used as a background sample to check how well we can reject CVs by their light-curve shapes. We decomposed the light curves of these multi-outburst CVs into 421 individual outbursts. The light curves of these outbursts were fit with microlensing models, and we found that none of them passed our selection criteria within $0.3<t_{\mathrm{E}}<2$ days. With zero false positive in 421 outbursts, Poisson statistics provides an upper limit of 2.3 at the $90 \%$ c.l.. Then, the expected $90 \%$ c.l. upper limit of contamination among the 197 single-outburst CVs in our sample is $2.3 \times 197 / 421=1.1$, or $11 \%$ of our ten $t_{\mathrm{E}}<2$ day events.

After the MOA event selection was complete, we extended the light curves by adding the 2008-2010 MOA data and found that none of the ten $t_{\mathrm{E}}<2$ day events have any other brightening episode. The MOA group also requested the OGLE light curves of these events. Seven of the ten short events were also observed by OGLE-III, the third phase of OGLE survey, and none of them have any other brightening in the 8-year OGLE-III light curves. Furthermore, the event MOA-ip-3 was also observed during OGLE-I and OGLE-II surveys and exhibits no further variations, which gives 16 years of coverage. Although the event MOA-ip-10 was not observed by OGLE-III, it was observed during OGLE-II survey in 1996-2000 and no brightening was seen. Finally, we checked the public database of the MACHO project, and found that the light curves of MOA-ip-3, MOA-ip-5, MOA-ip-6, and MOA-ip-8 had light curves with no photometric 
variation observed during the 1993-1999 observing seasons. These data support our conclusion that these short events are not CVs.

(4) Background supernovae: The dominant background in the microlensing survey toward the Large and Small Magellanic Clouds (LMC and SMC) was supernovae in galaxies at large distances behind the Magellanic Clouds, and methods to remove this background from the microlensing sample by means of their asymmetric light curves were developed $\underline{38}, \underline{45}, \underline{46}$.

The event rate of background supernovae in galactic bulge microlensing surveys is much smaller than that toward the Magellanic Clouds for the following reasons. The interstellar extinction toward the galactic bulge is much higher, $A_{V}=1-6 \mathrm{mag} \underset{47}{ }$, than towards the Magellanic Clouds with $A_{V} \sim 0.3 \mathrm{mag} \underline{48}$. Also, the LMC is $\sim 6.25$ times more distant than the galactic bulge, and the SMC is even more distant, so the Magellanic Could source stars tend to be $\sim 2$ mag fainter. The ratio of supernovae to microlensing events is also dramatically reduced by the factor of $\sim 100$ ratio between the high bulge microlensing rate $\frac{17,18,19,39}{3}$ and the low rate towards the Magellanic Clouds $38, \underline{40,46}$.

Although supernovae do not repeat like CVs, the shape of supernova light curves is somewhat similar to those of CVs. Both tend to be highly asymmetric with a steep rise and a slow decline. So that they can be rejected by the microlensing fits $38,45,46$ in the same way as we have rejected CVs.

Although supernovae do not pass our microlensing selection criteria, they could not contaminate our short timescale event sample even if they did, as they have timescales of $\sim 30$ days or longer.

(5) Binary microlensing events: Binary lens systems generate small caustics when the separation of the lens masses is either much smaller or much larger than the Einstein radius. Wide binary events, however, can only mimic a short timescale single lens event when one of the lens masses has a low mass $\underline{13} \underline{49}$. So, wide binaries are not really a background, and we consider them in $\S 8$ where we consider the distinction between very wide and free-floating planets. The $2-\sigma$ limits on the minimum separation to a host star are shown in Table 1 in the main paper. These limits are independent of the mass ratio and $R_{\mathrm{E}}$ value for the host star in the limit where $q \ll 1$.

The situation with close binaries is different, because close binaries have two small caustics far from the center of mass that can give rise short duration lensing events even when the masses of the lens objects are large. We have fit all ten of the $t_{\mathrm{E}}<2$ day events with close binary models and found that for most of the short events, a close-binary model is strongly excluded, being disfavored over the single-lens model by $\Delta \chi^{2} \geq 30$. The only exceptions are events with relatively poor sampling. For events MOA-ip-2 and MOA-ip-6, the close binary models are disfavored by $\Delta \chi^{2}=17.2$ and 9.8 , respectively. 
These imply formal probabilities of $<0.02 \%$ and $<0.8 \%$ for these events to be caused by close binaries.

Event MOA-ip-5 is the only event for which the close binary model cannot be rejected. The best fit model for this event is actually a planetary wide binary model, which has a $\chi^{2}$ value lower than the single lens $\chi^{2}$ value by $\Delta \chi^{2}=27.8$. However, this level of $\chi^{2}$ improvement is likely to be due to systematic photometry errors spread over a large number of data points, and we do not consider this a large enough $\chi^{2}$ improvement to indicate the detection of a host star. The best close binary model also gets some $\chi^{2}$ improvement from regions of the light curve that are distant from the peak shown in Fig. 1, and it has a $\chi^{2}$ value that is better than the best fit single lens light curve by $\Delta \chi^{2}=11.9$ with 4 more fit parameters (as the magnification of the single lens model is too low for finite source effects to be important). Formally, this would be significant at the $98 \%$ confidence level, but only $\Delta \chi^{2}=5.5$ of this difference comes from the region within 10 days of the light curve peak. A $\Delta \chi^{2}=5.5$ difference is only significant at the $\sim 70 \%$ confidence level, so we do not consider the close binary model to be be significantly favored for this event. The light curve coverage is insufficient to distinguish between the $t_{\mathrm{E}}<2$ day single lens and close binary models.

We can compare the case of MOA-ip-5 to the short events with better sampling in order to estimate the probability that MOA-ip- 5 is due to a close binary lens system and is not a short timescale event. This analysis has revealed the 9 other events listed in Table 1 in the main paper, 2 wide separation planetary (or brown dwarf) binary events and one close binary (Bennett et al. in preparation). There is one other short event that is a poor fit to a single lens event, but a worse fit to a close binary. (It is probably a CV.) Thus, there are $\sim 11$ short events that are best fit by single lens or wide binary models, but only one that are best fit by a close binary. So, it is unlikely that MOA-ip-5 is a close binary event. The probability that it is a close binary can be quantified in an analysis like that by Bennet ${ }^{50}$, but it is simplest to just assume that MOA-ip-5 is a single lens event.

We can also constrain the short event contamination due to close binaries by using the observed binary fraction and distribution. About $30 \%$ of main sequence stars have binary companions ${ }^{51}$. Only the small caustics due to close binaries with separation $0.05<d / R_{\mathrm{E}}<0.3$ in Einstein radius unit can resemble the short single-lens microlensing events. For $0.05>d / R_{\mathrm{E}}$, the caustics become too small to be detected. For $d / R_{\mathrm{E}}>0.3$, the effect of the host star is observable. This range corresponds $-1<\log (a / \mathrm{AU})<0$ for the typical lens systems, i.e., $\mathrm{K}$ or M-dwarfs. Only $6 \%$ of binaries have separation within this range $\underline{52,53}$. The event probability and detection efficiency for short events, which depend only on magnification timescale, are equivalent for close binary events and for the single lens events due to unbound or distant planetary mass objects. So, we can directly compare the number of close binary and single lens systems to estimate the contamination by close binaries. The fraction of short events due to close binaries versus single lens events is $0.3 \times 0.06 \times 2 / 1.8=0.02$. 
There is an additional factor of two for the close binaries because of the two small caustics generated a close binary lens system. This is consistent with our conclusion above that the contamination of our sample of short events by close binary lens systems is negligible.

(6) Microlensing by high velocity stars and Galactic halo stellar remnants. Stars with very high velocity could also produce short timescale events. The mean timescale of the events due to stars in the bulge is $t_{\mathrm{E}}=R_{\mathrm{E}} / v_{\mathrm{t}}=R_{\mathrm{E}} /\left(D_{1} \mu\right) \sim 20$ days, where $\mu=v_{\mathrm{t}} / D_{1}$ is the lens-source relative proper motion. So, it would require stars with at least an order of magnitude higher velocity than the typical stars to explain the events with $t_{\mathrm{E}} \leq 2$ days.

For lens stars with high velocity at fixed distance $D_{1}, t_{\mathrm{E}}$ is proportional to $\mu^{-1}$. The

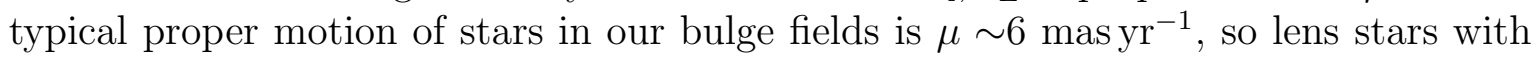
$\mu \geq 60$ mas yr$^{-1}$ can have $t_{\mathrm{E}} \leq 2$ days. However the number of such high proper motion stars with $\mu \geq 60$ mas yr $^{-1}$ are only $6 \times 10^{-5}$ of stars in the OGLE-II proper motion catalog in the galactic bulge fields ${ }^{\underline{54}}$. The event rate per lens star is proportional to the Einstein radius, $R_{\mathrm{E}}$, times a transverse velocity, $v_{\mathrm{t}}$, so the event rate due to the high proper motion stars with a typical mass of $0.3 M_{\odot}$ is a factor of $\sqrt{0.3 M_{\odot}} / \sqrt{0.001 M_{\odot}} \times$ $60 / 6=170$ higher than that of a Jupiter mass lens with typical kinematics. If we make the (unrealistic) assumption that all the high proper motion stars are high velocity stars in the bulge, then we can obtain the expected rate of $t_{\mathrm{E}} \leq 2$ day events due to these high velocity stars. This is simply the fraction of high proper motion stars times the difference in event rate, or $6 \times 10^{-5} \times 170=0.01$ per star. This compares directly to the inferred number, 1.8, of Jupiter mass lens objects from the main analysis. So, the background of high velocity stellar lenses is more than two orders of magnitude less than the signal. Moreover, a more straight forward interpretation of these high proper motion stars in the OGLE-II proper motion catalog is that they are just just nearby stars instead of high velocity stars. Microlensing events due to nearby high proper motion stars are already included in our simulations of the $t_{\mathrm{E}}$ distribution, so they do not produce the observed population of short $t_{\mathrm{E}}$ events. Thus, the above upper limit of 0.01 per star is a very conservative limit.

The conclusion that these high proper motion stars are nearby stars with regular kinematics instead of more distant high velocity stars are the following:

(a) The proper motion distribution in the OGLE-II catalog of the bulge follows a slope of $\log (N)=-3 \log (\mu)+$ const., without any distinct features, for $\mu>10$ mas yr$^{-1}$. This matches the expectation for a standard kinematical model and a uniform distribution of stars in space ${ }^{\underline{54}}$. This implies that most of these high proper motion stars are not high velocity stars, but are instead nearby stars within about $300 \mathrm{pc}$ which correspond the scale height of the disk. The proper motion of disk stars at a distance of $<300 \mathrm{pc}$ with velocity of $v \sim 30 \mathrm{~km} \mathrm{~s}^{-1}$ is $>20$ mas $\mathrm{yr}^{-1}$.

(b) Galactic bulge lens stars contribute about $70 \%$ of all events observed towards the 
bulge, and the typical bulge star velocity is about $120 \mathrm{~km} \mathrm{~s}^{-1}$. A high velocity star, with a velocity an order of magnitude higher than this, would have a velocity higher than the escape velocity from the Galaxy. Galactic bulge radial velocity observations found that no stars with radial velocity of $v_{\mathrm{l}}>400 \mathrm{~km} \mathrm{~s}^{-1}$ in a sample of 3200 stars and no significant deviations from a Gaussian distribution ${ }^{55}$. This confirms that there is no high velocity population in the bulge that could explain these short events.

These arguments indicate that there is no significant population of high velocity stars to explain our detection of short events.

A Galactic halo population of ancient stellar remnants, such as white dwarfs, neutron stars or black holes have sometimes been suggested as a explanation of the microlensing events seen towards the LMC by the MACHO Collaboration ${ }^{38}$. If so, their velocities should be $v \sim 200 \mathrm{~km} \mathrm{~s}^{-1}$. The typical $t_{\mathrm{E}}$ value due to the stellar remnants in the halo is $>20$ days, so to get events with $t_{\mathrm{E}} \leq 2$ days, the velocities must be an order of magnitude larger, $v \sim 2000 \mathrm{~km} \mathrm{~s}^{-1}$. So, like the stars considered above, a Galactic halo population of stellar remnants cannot explain these short events due to the Galactic escape velocity constraint.

A final test of the possibility of high velocity lenses could be made by the detection of finite source effects for short events with relatively high magnification. Microlensing events due to massive lenses (with large $R_{\mathrm{E}}$ ) and high velocities should show this effect much less frequently than events due to planetary mass lenses (with small $R_{\mathrm{E}}$ ) and more typical velocities. In the current sample, events ip-1 and ip-10, have magnification high enough so that they might possibly show finite source effects. A finite source model for event ip-1, yields an improvement of $\Delta \chi^{2}=1.6$ for a finite source model, which would imply a relative proper motion of $\mu \sim 6$ mas/yr, which is quite typical of bulge lensing events. For event ip-10, the best finite source model improves $\chi^{2}$ by only $\Delta \chi^{2}=0.1$, but the implied lower limit on the relative proper motion is only $\mu>4$ mas/yr, so most of the range of expected values is allowed.

We can expand this test with two short events from the MOA alert system that occurred too late to be in our statistical sample. Event MOA-2009-BLG-450 has $t_{\mathrm{E}}=1.2$ days and a peak magnification of 85. It shows no finite source effects, which implies a relative proper motion lower limits of $\mu>6$ mas/yr. MOA-2010-BLG-418 was one of the shortest events known, with $t_{\mathrm{E}}=0.41$ days and a peak magnification of 23 . It does show a 3- $\sigma$ signal for a finite source effect corresponding to a relative proper motion of $\mu=7$ mas $/ y r$. Thus, the event most sensitive to finite source effects does show them, and the other events have limits on $\mu$ which are consistent with expectations for a planetary mass population. 


\section{Detection Efficiency Simulations}

To compare observed $t_{\mathrm{E}}$ distribution with the model, $\Phi_{t_{\mathrm{E}}}$, we estimated the detection efficiency of our experiment using Monte Carlo simulations following previous work in the field ${ }^{14}$. Artificial microlensing events were added at random positions in the observed images, using PSFs derived from nearby stars in each field. The parameters of these artificial events were uniformly generated at random in the following ranges for the impact parameter, $u_{0}$, time of peak magnification, $t_{0}$, Einstein radius crossing time, $t_{\mathrm{E}}$, and source magnitude, $I$ : $0 \leq u_{0} \leq 1.5,2453824 \leq t_{0} \leq 2454420 \mathrm{JD}, 0.1 \leq t_{\mathrm{E}} \leq 250$ days, and $14.25 \leq I \leq 21.15$ mag. (The $t_{0}$ range is the range of observations in this data set.) The source magnitudes were weighted by the combined Luminosity function (LF) from MOA and the Hubble Space Telescope (HST) ${ }^{56}$. This uses the MOA LF for bright stars and HST for faint stars down to $I=24$. This combined LF is calibrated to the extinction Galactic bulge distance for each field using the position of red clump giant stars in the color magnitude diagram, as red clump giant stars serve as a good standard candle.

Once the images with artificial events were created, we processed them with the same analysis pipeline and selection criteria used in the analysis of the actual data. We evaluated our detection efficiency as a function of $t_{\mathrm{E}}$ in each field by simulating 20 million artificial events. The results are shown in Fig. 2, While the detection efficiencies drop sharply for decreasing $t_{\mathrm{E}}$ values, the detection efficiency is still significant at $t_{\mathrm{E}} \sim 1$ day, thanks to the high cadence of our survey. Fig. 3 shows the $t_{\mathrm{E}}$ distribution with the data corrected for the detection efficiencies. This is similar to Fig. 2 of the main paper, except that in Fig. 2, it is the predicted model $t_{\mathrm{E}}$ distributions that have been corrected for detection efficiencies. The flattening of the $t_{\mathrm{E}}$ distribution at $t_{\mathrm{E}} \lesssim 2$ days indicates a rising mass function very low (planetary) masses, because the intrinsic microlensing probability scales with the lens mass $M$ as $\sim \sqrt{M}$.

\section{$5 \quad$ Tests for Systematic Biases}

When light curve coverage is poor or the photometry errors are large, the light curve models can become degenerate. A model with a short timescale (small $t_{\mathrm{E}}$ ) and a large $u_{0}$ value can look quite similar to a longer event with a smaller $u_{0}$ value and a fainter source flux, $F_{\mathrm{s}}$. The degeneracy tends to be worse for faint source stars blended with brighter stars, and it can lead to a bias in the inferred event parameters. This bias has been well studied ${ }^{57}$. To avoid such systematic biases in the observed $t_{\mathrm{E}}$ distribution, we have rejected microlensing candidates with fit parameters that are not well constrained as described in Section 2. Note that the errors in $t_{\mathrm{E}}$ listed in Table 1 in the main paper include the correlation with other parameters. In order to check for possible systematic parameter biases and significant contamination of our event sample, we have conducted several consistency checks with the observed and simulated events.

The distribution of the impact parameter, $u_{0}$ which is purely geometric, can be used 
as a test that the sample is dominated by real microlensing events. Fig. 4 compares the observed and simulated $u_{0}$ distributions for events in different $t_{\mathrm{E}}$ ranges. We performed the Kolmogorov-Smirnov test, which yields probabilities of $77 \%, 76 \%, 51 \%, 97 \%$ and $74 \%$ for the ranges $t_{\mathrm{E}}=0.3-2.0,2.0-13,13-69,89-200$ days and the full sample, respectively. All the sub-samples are consistent with the simulated distributions, so there is no evidence of significant contamination by intrinsic variable stars in the $u_{0}$ distributions.

We also compared the input (true) $t_{\mathrm{E} \text {,in }}$ values input into our event simulations to the $t_{\mathrm{E}, \text { out }}$ values output by the light curve analysis. The results are shown in Fig. 5 , This test shows that the $t_{\mathrm{E} \text {,out }}$ values are a reasonably accurate representation of the input $t_{\mathrm{E} \text {,in }}$ values, but that there is a small systematic offset that makes the mean $t_{\mathrm{E} \text {,out }}$ systematically larger than the input (true) $t_{\mathrm{E} \text {,in }}$ values, independent of the value of $t_{\mathrm{E} \text {,in }}$. This implies that the bias in our measured $t_{\mathrm{E}}$ is too small to influence our conclusions. Furthermore, the sign of the small bias that we do see implies that we might be slightly underestimating the number of short $t_{\mathrm{E}}$ events.

\section{Likelihood Analysis of the Substellar and Stellar Mass Functions}

The Einstein radius crossing time $t_{\mathrm{E}}$ is the only observable in the regular single lens microlensing event and is given by Equation (11). Although the physical parameters of the lens and source are degenerate, a model $t_{\mathrm{E}}$ distribution, $\Phi\left(t_{\mathrm{E}}\right)$, can be calculated using a Monte Carlo simulation for an assumed mass function with a standard galactic mass density and velocity model $\underline{20}$. The same method have been applied in the previous studies 14,17 of the stellar mass function in the Galactic bulge.

We used a Bayesian likelihood analysis to determine the mass function model parameters for both the power-law and log-normal mass functions. For the stellar and brown dwarf mass functions this was done both with and without the events with $t_{\mathrm{E}} \leq 2$ day events, and we found that the differences in the implied stellar and brown dwarf mass functions was negligible, as shown in Table 3. Fig. 6] shows the likelihood distribution for the mass function slope in the brown dwarf regime, $\alpha_{3}$, for the power-law model. This analysis finds $\alpha_{3}=-0.48_{-0.29}^{+0.37}$, which is slightly higher than, but consistent with, previous estimates of $\alpha_{3}=0-0.358,59,60$. Note that this $\alpha_{3}$ values does not change when we consider additional mass function models in the following section.

Fig. 7 is a contour plot of the likelihood distribution for the log-normal mass function parameters $M_{\mathrm{c}}$ and $\sigma_{\mathrm{c}}$. The contours indicate the $68 \%$ and $95 \%$ confidence intervals and the cross indicates the maximum of the two-dimensional likelihood distribution. The log-normal parameter values determined by this analysis are a mean mass $M_{\mathrm{c}}=0.12 \pm 0.03$ and a log-normal width of $\sigma_{\mathrm{c}}=0.76_{-0.16}^{+0.27}$. These are consistent with the values of $M_{\mathrm{c}}=0.079_{-0.016}^{+0.021}$ and $\sigma_{\mathrm{c}}=0.69_{-0.01}^{+0.05}$ determined in the previous work ${ }^{22}$.

The power-law and log-normal models indicate that the number of brown dwarfs is $0.73_{-0.19}^{+0.22}$ and $0.70_{-0.30}^{+0.19}$ (respectively) times the number of main sequence stars in the mass 
range $0.08 \leq M / M_{\odot} \leq 1.0$, which we denote by $N_{*}$. These values are higher than the value of 0.2 for the model (3) in Section 7.1. The best model $t_{\mathrm{E}}$ distributions, $\Phi\left(t_{\mathrm{E}}\right)$, are indicated as dashed lines in red and blue for the power-law and log-normal models, respectively, in Fig. 2 of the main paper and Fig. 3. As one can see, the both models represent data very well for $t_{\mathrm{E}} \geq 2$, but there is a significant excess at $t_{\mathrm{E}} \leq 2$ days. For $t_{\mathrm{E}} \leq 2$ days, we have detected 10 events compared to the predictions of 1.5 and 2.5 events without the planetary mass population for the power-law and log-normal models, respectively. The Poisson probability of detecting 10 events when only 1.5 or 2.5 events are predicted are $4 \times 10^{-6}$ and $3 \times 10^{-4}$ for the power-law and log-normal models, respectively. At $t_{\mathrm{E}} \leq 1$ days, we observe 3 events compared to predictions of 0.12 and 0.24 events for the planet-free power-law and log-normal models, respectively. The contributions of the best fit planetary mass distributions are indicated by the red and blue dotted curves for the power-law and log-normal mass function respectively, and the full distributions including lens objects of all masses are given by the red and blue solid curves. Both models provide an excellent fit to the observed $t_{\mathrm{E}}$ distribution.

\section{Likelihood Analysis with the Other Mass Functions}

In this section we present the likelihood analysis for mass functions that differ from the models (1) and (2) shown in the main paper. Section 7.1 presents the results for an alternative stellar and brown dwarf mass function that is discontinuous near the brown dwarf-stellar boundary, and in Section 7.2 we consider a planetary power-law mass function. This is likely to be more realistic than the $\delta$-function model, but its parameters are difficult to measure with the current data set.

\subsection{Discontinuous Substellar Mass Function}

In the main paper, we used two models, (1) the power-law mass function (MF) measured in the galactic bulge $\underline{21}$ with a continuous brown dwarf MF that was fit to the observed $t_{\mathrm{E}}$ distribution, and (2) a log-normal MF fitted to the data, with best fit parameters are consistent with the values found with previous work ${ }^{22}$.

Here, we present the likelihood analysis with model (3), a discontinuous MF 24,61 which uses the universal Galactic IMF, $\psi$, based on the average of various stellar clusters $\frac{62}{}$ with $\alpha_{1}=2.3$ for $0.5 M_{\odot}<M, \alpha_{2}=1.3$ for $0.075 M_{\odot}<M<0.5 M_{\odot}$, and $\alpha_{3}=0.3$ for $0.01 M_{\odot}<$ $M<0.075 M_{\odot}$. But this $\mathrm{MF}$ is discontinuous at the hydrogen burning limit $M_{\mathrm{HBL}}=$ $0.075 M_{\odot}$, with a discontinuity of a factor of $R_{\mathrm{HBL}}=\psi_{\alpha_{3}}\left(M_{\mathrm{HBL}}\right) / \psi_{\alpha_{2}}\left(M_{\mathrm{HBL}}\right)=0.2-0.3$. We assume that stars heavier than $1 M_{\odot}$ have evolved to become stellar remnants, as we have done with the other mass functions. The steeper slope, $\alpha_{1}=2.3$, implies fewer massive stellar remnants. In the planetary regime, we use a $\delta$-function MF just as with models (1) and (2). Fig. 8 shows the result of likelihood analysis for $R_{\mathrm{HBL}}=0.3$. The resulting 
parameters are $M / M_{\odot}=1.9_{-0.9}^{+1.4} \times 10^{-3}$ and $\Phi=0.50_{-0.10}^{+0.11}$ as shown in Table 3 . In this model, planetary mass objects with mass of $1.9_{-0.9}^{+1.4}$ Jupiter mass are $1.3_{-0.4}^{+0.7}$ times as frequent as main sequence stars. With a slightly stronger mass function discontinuity, $R_{\mathrm{HBL}}=0.2$, we find similar values: $M / M_{\odot}=2.2_{-1.0}^{+1.6} \times 10^{-3}$ and $\Phi=0.51_{-0.10}^{+0.10}$. Fig. 9] presents the best fit $t_{\mathrm{E}}$ distribution for the case of $R_{\mathrm{HBL}}=0.3$. One can see that this model generates a narrower $t_{\mathrm{E}}$ distribution. It has fewer long $(\geq 40$ day) and short ( $\leq 10$ day) events compared to the peak ( $\sim 20$ days), than models (1) and (2) shown in the main paper. This is because this model has a steeper power law for high masses, $0.5 M_{\odot}<M$, and fewer brown dwarfs. This model provides a worse fit to the data than models (1) and (2), with a maximum likelihood value that is a factor of 25 worse than model (1). However,the discontinuous MF model (3) does have one fewer model parameter that is fit to the data and it employs a mass function at the high end, which provided a worse fit to long timescale events in previous studies $\underline{14}, \underline{63}$. Since the relatively poor fit at the high mass end contributes to the poor likelihood value, we consider this model of the mass function of low-mass objects to be acceptable. The main conclusion, however, is that this mass function does not alter our conclusion that a new population of planetary mass objects is needed to explain the data, although it does imply a slightly higher mass for these objects.

\subsection{Power-law Planetary Mass Function}

In the main paper, we used a $\delta$-function mass function for the planetary mass objects, but this is not likely to be realistic. Exoplanets are known to span a wide range of masses, and lower mass planets are much more easily ejected from their host stars in 2-body encounters. So, we have also considered a power-law form for the planetary part of the mass function, with an index of $\alpha_{\mathrm{PL}}$ for the mass range $10^{-5} \leq M / M_{\odot} \leq 0.01$. This is assumed to be continuous with the power-law stellar and substellar mass function of MF model (1) in the main paper, at $M=0.01 M_{\odot}$. Our result does not depend on the lower mass limit $10^{-5} M_{\odot}$, because our sensitivity to events with $M<10^{-4} M_{\odot}$ is quite small, due to the very low the detection efficiency at small $t_{\mathrm{E}}$. The power-law index, $\alpha_{3}$, for the brown dwarf mass regime, $0.01 \leq M / M_{\odot} \leq 0.08$, was fit with $\alpha_{\mathrm{PL}}$, simultaneously.

Fig. 10 shows the likelihood function for $\alpha_{3}$ and $\alpha_{\mathrm{PL}}$, and the resulting best fit parameters are $\alpha_{3}=0.49_{-0.27}^{+0.24}$ and $\alpha_{\mathrm{PL}}=1.3_{-0.4}^{+0.3}$. This $\alpha_{3}$ value is consistent with our result for MF model (1) with and without $\delta$-function planetary mass function (see Table 3). The value $\alpha_{\mathrm{PL}}$, which is as steep as $\alpha_{2}$, is much steeper than $\alpha_{3}$, indicating that this planetary mass objects are separate population from the brown dwarfs. This model also predicts a larger, but poorly constrained, number of planetary mass objects per star with $N_{\mathrm{PL}} / N_{*}=5.5_{-4.3}^{+18.1}$, due to our poor sensitivity to very low-mass lenses.

Fig. 11 shows the best fit $t_{\mathrm{E}}$ distribution compared with data and the best fit model (1) in the main paper for a comparison. Although this power-law planetary mass function model has a maximum likelihood that is a factor of 0.75 smaller than model (1), it has one fewer model parameter, so formally, it is a slightly better fit. This implies that the current data set is not able to constrain the shape of the mass function at sub-Jupiter masses. 


\section{Relative Frequencies of Unbound/Distant and Bound Planets}

Our sample includes three previously known events in which a planet and its host star are both detected: MOA-2007-BLG-192 ${ }^{64,65}$, OGLE-2007-BLG-368ํㅗㄹ , and OGLE-2007-BLG$349^{31}$.

The short timescale events caused by planets bound to host stars with a separation of $<10-20 \mathrm{AU}$ are detected as binary lens systems with a very small mass ratio. The events in our sample are detected as single lens events, because the planetary mass lenses are sufficiently isolated from any host star that the microlensing effect of the host star cannot be seen. These isolated planetary mass lenses can be either unbound or in distant orbits about a host star, and they can be distinguished from planetary mass lenses detected in binary events in two possible ways. The binary lens systems reveal the presence of a nearby host star by showing the effect of a planetary caustic in their light curves or by showing a low-magnification bump due to lensing by the host star $25,66,67$. We searched for the signature of host stars in our short events by fitting binary light curves to the data and found that none of the 10 selected events with $t_{\mathrm{E}}<2$ days shows any significant evidence of host stars (see bellow for their limits). However, our analysis has revealed three short events, which fail our selection criteria, that have clear binary lens caustic crossing features, as well as a very low amplitude signal due to lensing by the host star. Detailed modeling of these 3 events indicates wide binary models with lens mass ratios of $q<0.05$ for two of these events and a close binary for the third (Bennett et al. in preparation).

Thus, the total number of events in this sample in which both a planet and its host star are detected is 5. However, for two of these events, MOA-2007-BLG-192 and OGLE-2007BLG-349, the planet is detected via light curve features due to the central caustic. This means that these planets have been detected only because of the gravitational effect of the host stars. For the other 3 planet plus host star events, the planet would have been detected without the influence of the host stars. Of course, there are likely to be some planets even closer to their host stars that are undetectable (by microlensing) because of the presence of the host star, so it is reasonable to presume that there are 5 planetary mass objects in binary lens systems in this sample that were detected with approximately the same efficiency as the planetary mass objects detected as isolated lens systems. This allows us to estimate the ratio of the new population of unbound or distant planetary mass objects to the planets found with host stars. The power-law mass function implies 11 isolated planetary mass lenses (that comprise our unbound or distant planetary mass sample) in our full event sample, so this ratio is $11 / 5=2.2$. With the log-normal mass function, the number of events due to planetary mass objects is $\sim 11$, but 3 of these are from the low-mass tail of the brown dwarf mass function. This leaves 8 unbound or distant planetary mass objects for a ratio to the planets with detectable host stars of $8 / 5=1.6$.

The frequency of planets per star was measured by microlensing 31 for the planet-to-star mass-ratio interval $-4.5<\log q<-2$ and separation $a \sim 2-8 \mathrm{AU}$, to be $d^{2} N_{\mathrm{pl}}(d \log q d \log s)^{-1}=$ 
$0.36 \pm 0.15$. This indicates $N_{\mathrm{pl}} \sim 0.9$ for this $q$ range and interval $a=1-10$ AU. Radial velocity (RV) surveys have found that $11.3 \%$ of stars have planets with $M \sin i>10 M_{\oplus}$ with periods less than 50 days 29 . The RV planets have periods that go down to about 3 days before the numbers really drop. So, we consider the RV survey to cover the period range $3<P<50$ day. If we assume a typical host star mass of 0.5 solar masses, then periods of $P=3$ and 50 days imply semi-major axes of $a=0.032$ and $0.21 \mathrm{AU}$, respectively, corresponding a range of 0.81 in $\log (a)$. Assuming a uniform planet distribution in $\log (a)$, we have a 0.21 bound planets per star between $a=0.21 \mathrm{AU}$ and $1 \mathrm{AU}$ where the microlensing numbers take over, corresponding a range of 0.68 in $\log (a)$. If we assume a planet distribution in period of $\mathrm{d} N_{\mathrm{pl}}(\mathrm{d} \log P)^{-1} \propto P^{0.26}$ as measured by RV for $\mathrm{G}$ dwarfs $\frac{68}{}$, we have a 0.29 bound planets per star. Thus, we have a total of 1.13-1.19 bound planets per star in the semi-major axis range $a=0.03-10 \mathrm{AU}$. This implies 1.5-1.6 times as many planetary mass objects that serve as isolated lens systems to planets known to orbit stars. This result is nearly identical to the microlensing-only data based argument above.

By necessity, we define our sample of isolated planetary mass lens objects as ones who don't have a host star that can be detected by microlensing, but this does not necessarily imply that host stars do not exist, which is why we interpret them as unbound or distant planets. The lower limit on the possible separation of a host star depends primarily on the magnification of the observed short timescale event. These minimum host star separations, $d_{\text {min }}$, are given in terms of the Einstein radius of the hypothetical host star, and the values for each of our $t_{\mathrm{E}} \leq 2$ day events are listed in the last column of Table 1 in the main paper. These are limits on the projected separation, and the typical semi-major axis corresponding to a host star with a separation of $d_{\text {min }}$ of one of our detected unbound or distant planetary mass object is $\sim(3 \mathrm{AU}) d_{\min }$, and therefore the typical lower limit on the separation of a host star is $\sim 14 \mathrm{AU}$, with a range of $\sim 7-45 \mathrm{AU}$ depending on the event.

Our data do not indicate what fraction of this population of unbound or distant planetary mass objects actually have host stars outside the region of detectability, but we can use the limits from direct detection searches to estimate this fraction. The Gemini Planet Imager ${ }^{9}$ has set upper limits on the number of stars with Jupiter-mass planets at semimajor axes of 10-500 AU, with the tightest limits of $<30 \%$ of stars with Jupiter-mass planets with semi-major axes in the range 50-250 AU. Based on these results, we estimated that the fraction of stars with a 1 Jupiter-mass planet with semi-major axes in the range $10 \mathrm{AU}<a<500 \mathrm{AU}$ is less than $40 \%$ assuming a uniform distribution in $\log a$. Since the new unbound or distant planetary mass population is comprised of $\sim 1.8$ times as many Jupiter-mass planets as stars, this comparison with the direct detection limits suggests that at least $75 \%$ of these unbound or distant planetary mass objects are not bound to any host star.

Note that our likelihood analysis of the mass function in the planetary mass range, shown in Fig. 8, givens a $1-\sigma$ lower bound on the $\delta$-function mass of the planetary mass objects of approximately a Saturn mass. The direct imaging results don't rule out Saturn mass planets. So, if the majority of the lens objects in the newly discovery planetary mass population have a mass of order that of Saturn or less, then the direct detection limits do 
not apply, and the majority of this new sample can be planets bound to host stars in wide orbits with semi-major axes of $a \gtrsim 7-45$ AU depending on the event. 


\section{References}

[1] Mayor, M., \& Queloz, D. A Jupiter-mass companion to a solar-type star. Nature 378, 355-359 (1995).

[2] Schneider, J. The extrasolar Planets Encyclopedia. http://exoplanet.eu/ (2011).

[3] Gaudi, B.S. Exoplanetary Microlensing, preprint at arXiv:1002.0332 (2010).

[4] Sumi, T. et al. A Cold Neptune-Mass Planet OGLE-2007-BLG-368Lb: Cold Neptunes Are Common. Astrophys. J. 710, 1641-1653 (2010)

[5] Zapatero Osorio, M. R., et al. Discovery of Young, Isolated Planetary Mass Objects in the $\sigma$ Orionis Star Cluster. Science 290, 103-107 (2000).

[6] Burgess, A. S. M. et al. Young T-dwarf candidates in IC 348. Astron. Astrophys. 508, 823-831 (2009)

[7] Quanz, S. P. et al. Search for Very Low-Mass Brown Dwarfs and Free-Floating PlanetaryMass Objects in Taurus. Astrophys. J. 708, 770-784 (2010).

[8] Marsh, K. A. et al. Deep Near-infrared Imaging of the Oph cloud core: Clues to the Origin of the Lowest-mass Brown Dwarfs. Astrophys. J. 719, 550-560 (2010).

[9] Lafrenière, D. et al. The Gemini Deep Planet Survey. Astrophys. J. 670, 1367-1390 (2007)

[10] Paczyński, B. Gravitational microlensing by the galactic halo. Astrophys. J. 304, 1-5 (1986)

[11] Liebes, S. Gravitational Lenses. Physical Review 133, 835-844 (1964)

[12] Bennett, D.P. et al. Planetary Microlensing from the MACHO Project, ASP Conf. Proc. 119: Planets Beyond the Solar System and the Next Generation of Space Missions, D.R. Soderblom, ed., 95 (1997).

[13] Di Stefano, R., \& Scalzo, R. A. A New Channel for the Detection of Planetary Systems through Microlensing. I. Isolated Events due to Planet Lenses. Astrophys. J. 512, 564-578 (1999)

[14] Sumi, T. et al. Microlensing Optical Depth toward the Galactic Bulge from Microlensing Observations in Astrophysics Group Observations during 2000 with Difference Image Analysis. Astrophys. J. 591, 204-227 (2003)

[15] Udalski, A. The Optical Gravitational Lensing Experiment. Real Time Data Analysis Systems in the OGLE-III Survey. Acta Astronomica 53, 291-305 (2003). 
[16] Udalski, A. et al. The Optical Gravitational Lensing Experiment. The Optical Depth to Gravitational Microlensing in the Direction of the Galactic Bulge. Acta Astronomica 344, 165-189 (1994)

[17] Alcock, C. et al. The MACHO Project: Microlensing Optical Depth toward the Galactic Bulge from Difference Image Analysis. Astrophys. J. 541, 734-766 (2000)

[18] Hamadache,C., et al. Galactic Bulge microlensing optical depth from EROS-2. Astron. Astrophys. 454, 185-199 (2006)

[19] Sumi, T. et al. Microlensing Optical Depth toward the Galactic Bulge Using Bright Sources from OGLE-II. Astrophys. J. 636, 240-260 (2006)

[20] Han, C. \& Gould, A. Stellar Contribution to the Galactic Bulge Microlensing Optical Depth. Astrophys. J., 592, 172-175 (2003)

[21] Zoccali, M., et al. The Initial Mass Function of the Galactic Bulge down to $\sim 0.15 \mathrm{M}_{\odot}$ Astrophys. J. 530, 418-428 (2000)

[22] Chabrier, G. Galactic Stellar and Substellar Initial Mass Function. Pub. Astron. Soc. Pacific 115, 763-795 (2003)

[23] Gould, A. Measuring the Remnant Mass Function of the Galactic Bulge. Astrophys. J. 535, 928 (2000).

[24] Thies, I. \& Kroupa, P. A Discontinuity in the Low-Mass Initial Mass Function. Astrophys. J. 671, 767-780 (2007)

[25] Han, C., \& Kang, Y. W. Probing the Spatial Distribution of Extrasolar Planets with Gravitational Microlensing. Astrophys. J. 596, 1320-1326 (2003)

[26] Veras, D., Crepp, J. R. \& Ford, E. B. Formation, Survival, and Detectability of Planets Beyond 100 AU. Astrophys. J. 696, 1600-1611 (2009).

[27] Marois, C. et al. Direct Imaging of Multiple Planets Orbiting the Star HR 8799. Science 322, 1348-1352 (2008).

[28] Winn, J.N., et al. Hot Stars with Hot Jupiters Have High Obliquities. Astrophys. J. 718, L145 (2010).

[29] Howard, A.W. et al. The Occurrence and Mass Distribution of Close-in Super-Earths, Neptunes, and Jupiters. Science 330, 653-655 (2010).

[30] Mao, S., \& Paczyński, B. Gravitational microlensing by double stars and planetary systems. Astrophys. J. 374, L37-L40 (1991) 
[31] Gould, A., et al. Frequency of Solar-like Systems and of Ice and Gas Giants Beyond the Snow Line from High-magnification Microlensing Events in 2005-2008. Astrophys. J. 720, 1073-1089 (2010)

[32] Bond, I. A., et al. Real-time difference imaging analysis of MOA Galactic bulge observations during 2000. Mon. Not. R. Astron. Soc., 327, 868-880 (2001)

[33] Tomaney, A.B. \& Crotts, A.P.S., Expanding the Realm of Microlensing Surveys with Difference Image Photometry. Astron. J. 112, 2872-2895 (1996)

[34] Alard, C. \& Lupton,R.H. A Method for Optimal Image Subtraction. Astrophys. J. 503, 325-331 (1998)

[35] Alard, C. Image subtraction using a space-varying kernel. Astron. Astrophys. Suppl. 144, 363-370 (2000)

[36] Schechter, P. L., Mateo, M., \& Saha, A. DOPHOT, a CCD photometry program: Description and tests. Pub. Astron. Soc. Pacific 105, 1342-1353 (1993)

[37] Udalski, A. et al. The Optical Gravitational Lensing Experiment. BVI Maps of Dense Stellar Regions. III. The Galactic Bulge. Acta Astronomica 52, 217-240 (2002)

[38] Alcock, C. et al. The MACHO Project: Microlensing Results from 5.7 Years of Large Magellanic Cloud Observations. Astrophys. J., 542, 281-307 (2000)

[39] Popowski, P., et al. Microlensing Optical Depth toward the Galactic Bulge Using Clump Giants from the MACHO Survey. Astrophys. J. 631, 879-905 (2005)

[40] Wyrzykowski, L., et al. The OGLE view of microlensing towards the Magellanic Clouds - I. A trickle of events in the OGLE-II LMC data. Mon. Not. R. Astron. Soc. 397, 1228$1242(2009)$

[41] Stetson, P. B. DAOPHOT - A computer program for crowded-field stellar photometry. Pub. Astron. Soc. Pacific 99, 191-222 (1987)

[42] Sahu, K. C. et al. Gravitational microlensing by low-mass objects in the globular cluster M22. Nature 411, 1022-1024 (2001)

[43] Sahu, K. C., Anderson, J. \& King, I. R. A Reexamination of the "Planetary" Lensing Events in M22. Astrophys. J. 565, L21-L24 (2002)

[44] Warner, B. Cataclysmic Variable Stars, by Brian Warner, pp. 592. ISBN 052154209X. Cambridge, UK: Cambridge University Press, (2003)

[45] Rest, A. et al. First Results from SuperMacho. American Astronomical Society Meeting 203, \#112.28; Bulletin of the American Astronomical Society 35, 1389 (2003) 
[46] Tisserand, P. et al. Limits on the Macho content of the Galactic Halo from the EROS-2 Survey of the Magellanic Cloud. Astron. Astrophys. 469, 387-404 (2007)

[47] Sumi, T. Extinction map of the Galactic centre: OGLE-II Galactic bulge fields. Mon. Not. R. Astron. Soc. 349, 193-204 (2004)

[48] Bessell, M. S. Concerning the reddening of the Magellanic Clouds. Astron. Astrophys., 242, L17-L20 (1991)

[49] Di Stefano, R., \& Scalzo, R. A. A New Channel for the Detection of Planetary Systems through Microlensing. II. Repeating Events. Astrophys. J. 512, 579-600 (1999)

[50] Bennett, D. P. Large Magellanic Cloud Microlensing Optical Depth with Imperfect Event Selection. Astrophys. J. 633, 906-913 (2005)

[51] Lada, C. J. Stellar Multiplicity and the Initial Mass Function: Most Stars Are Single. Astrophys. J. 640, L63-L66 (2006)

[52] Duquennoy, A., \& Mayor, M. Multiplicity among solar-type stars in the solar neighborhood. II - Distribution of the orbital elements in an unbiased sample. Astron. Astrophys. 248, 485-524 (1991)

[53] Delfosse, X, et al. M dwarfs binaries: Results from accurate radial velocities and high angular resolution observations. ASP Conference Series 318, 166-174 (2004)

[54] Sumi, T. et al. The Optical Gravitational Lensing Experiment: catalogue of stellar proper motions in the OGLE-II Galactic bulge fields. Mon. Not. R. Astron. Soc. 348, 1439-1450 (2004)

[55] Howard, C. D., et al. The Bulge Radial Velocity Assay (BRAVA). I. Sample Selection and a Rotation Curve. Astrophys. J. 688, 1060-1077 (2008)

[56] Holtzman, J. A. et al. The Luminosity Function and Initial Mass Function in the Galactic Bulge. Astrophys. J. 115, 1946-1957 (1998)

[57] Smith, M. C., Woźniak P. R., Mao, S., \& Sumi, T. Blending in gravitational microlensing experiments: source confusion and related systematics. Mon. Not. R. Astron. Soc. 380, 805-818 (2007)

[58] Cruz, K. L., et al. Meeting the Cool Neighbors. IX. The Luminosity Function of M7-L8 Ultracool Dwarfs in the Field. Astron. J. 133, 439-467 (2007).

[59] Metchev, S. A., Kirkpatrick, J. D., Berriman, G. B. \& Looper, D. A Cross-Match of 2MASS and SDSS: Newly Found L and T Dwarfs and an Estimate of the Space Density of T Dwarfs. Astrophys. J. 676, 1281-1306 (2008) 
[60] Reylí, C. et al. The ultracool-field dwarf luminosity-function and space density from the Canada-France Brown Dwarf Survey. Astron. Astrophys. 522, 112 (2010).

[61] Thies, I. \& Kroupa, P. A discontinuity in the low-mass IMF - the case of high multiplicity. Mon. Not. R. Astron. Soc. 390, 1200-1206 (2008)

[62] Kroupa, P. On the variation of the initial mass function. Mon. Not. R. Astron. Soc. 322, 231-246 (2001)

[63] Bennett, D. P. et al., Gravitational Microlensing Events Due to Stellar Mass Black Holes, Astrophys. J. 579, 639659 (2002)

[64] Bennett, D. P. et al., A Low-Mass Planet with a Possible Sub-Stellar-Mass Host in Microlensing Event MOA-2007-BLG-192. Astrophys. J. 684, 663-683 (2008)

[65] Kubas, D., et al. A frozen super-Earth orbiting a star at the bottom of the Main Sequence. Astron. Astrophys., submitted (arXiv:1009.5665) (2010)

[66] Han, C., Gaudi, B. S., An, J. H. \& Gould, A. Microlensing Detection and Characterization of Wide-Separation Planets. Astrophys. J. 618, 962-972 (2005)

[67] Han, C. Characterization of Microlensing Planets with Moderately Wide Separations. Astrophys. J. 700, 945-948 (2009)

[68] Cumming, A., Butler, R. P., Marcy, G. W., Vogt, S. S., Wright, J. T., \& Fischer, D. A. The Keck Planet Search: Detectability and the Minimum Mass and Orbital Period Distribution of Extrasolar Planets. Pub. Astron. Soc. Pacific 120, 531-554 (2008) 

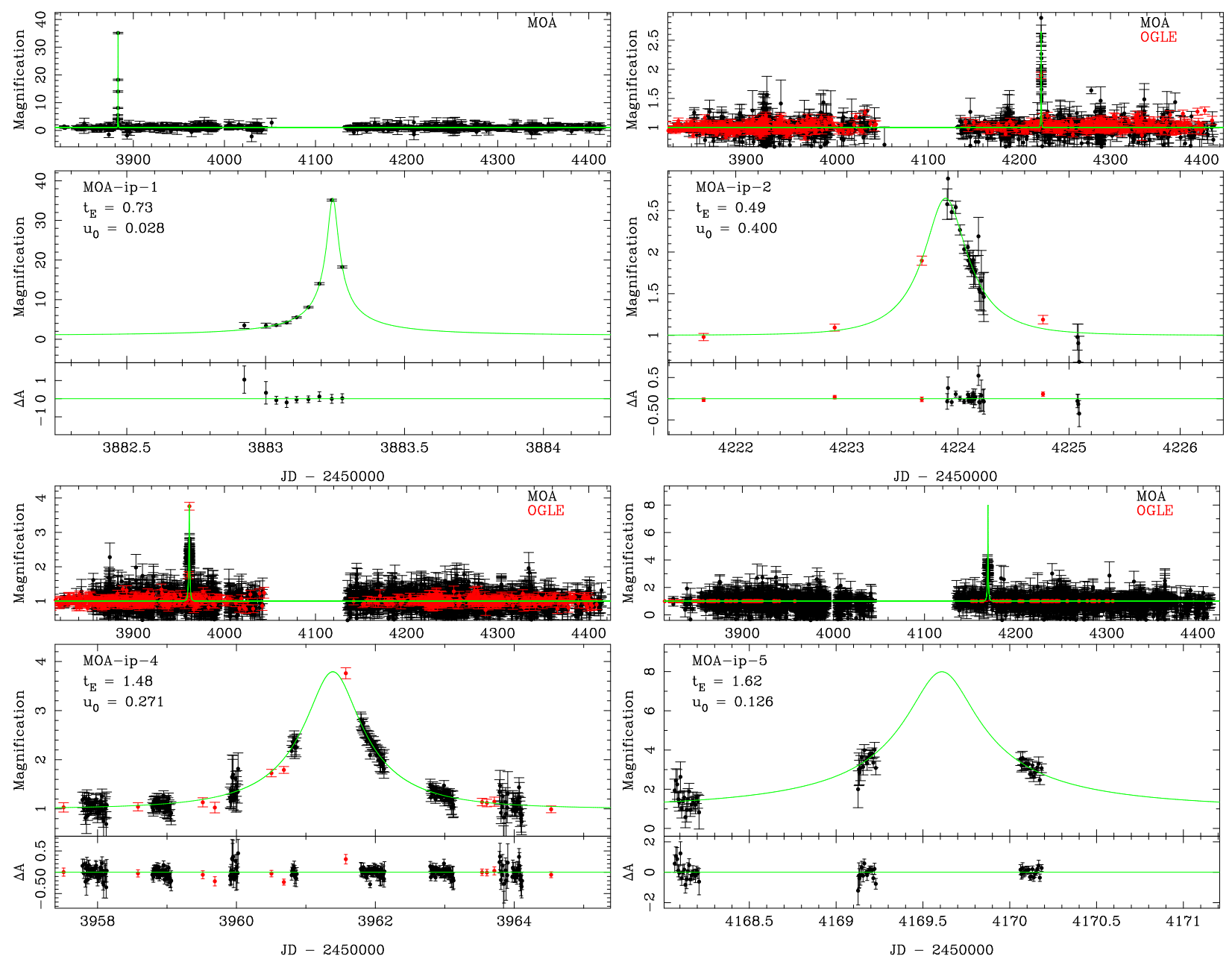

Figure 1. - Light curves of the 8 short microlensing events with $t_{\mathrm{E}} \leq 2$ days that are not shown in the main paper. MOA data are in black and OGLE data are in red with error bars indicating s.e.m. For each event, the upper panel shows the full two-year light curve, and the middle and lower panels show a zoom-in around the peak and the residual from the best fit model, respectively. Although the light curve coverage of event MOA-ip-5 is somewhat sparse, it has 69 data points during the peak $\left(t_{0} \pm t_{\mathrm{E}}\right)$, the second largest number in our short event sample (see Table 1 in the main paper) and our light curve model fits support the conclusion that this is a microlensing event. 

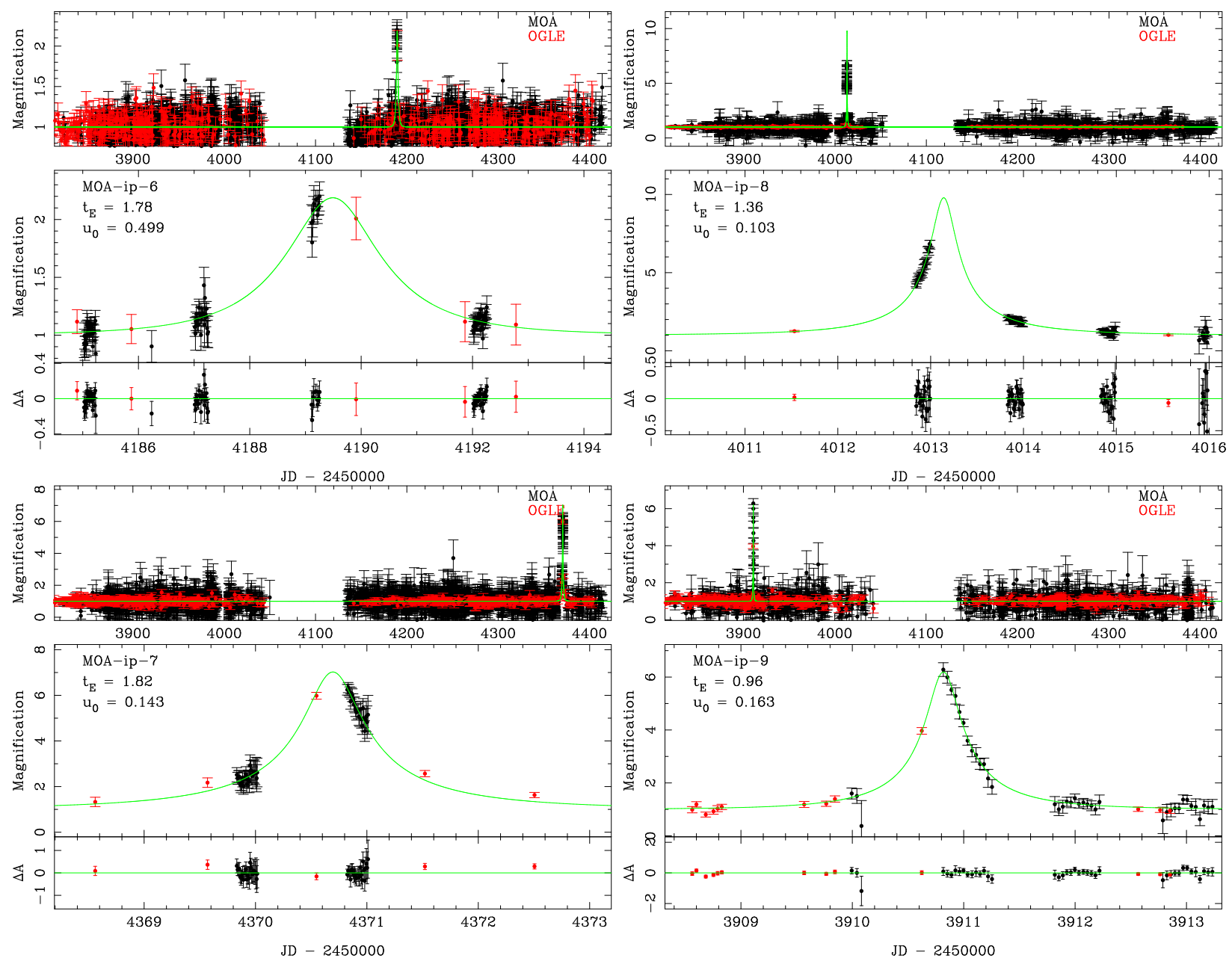

Figure 1.- continued. 


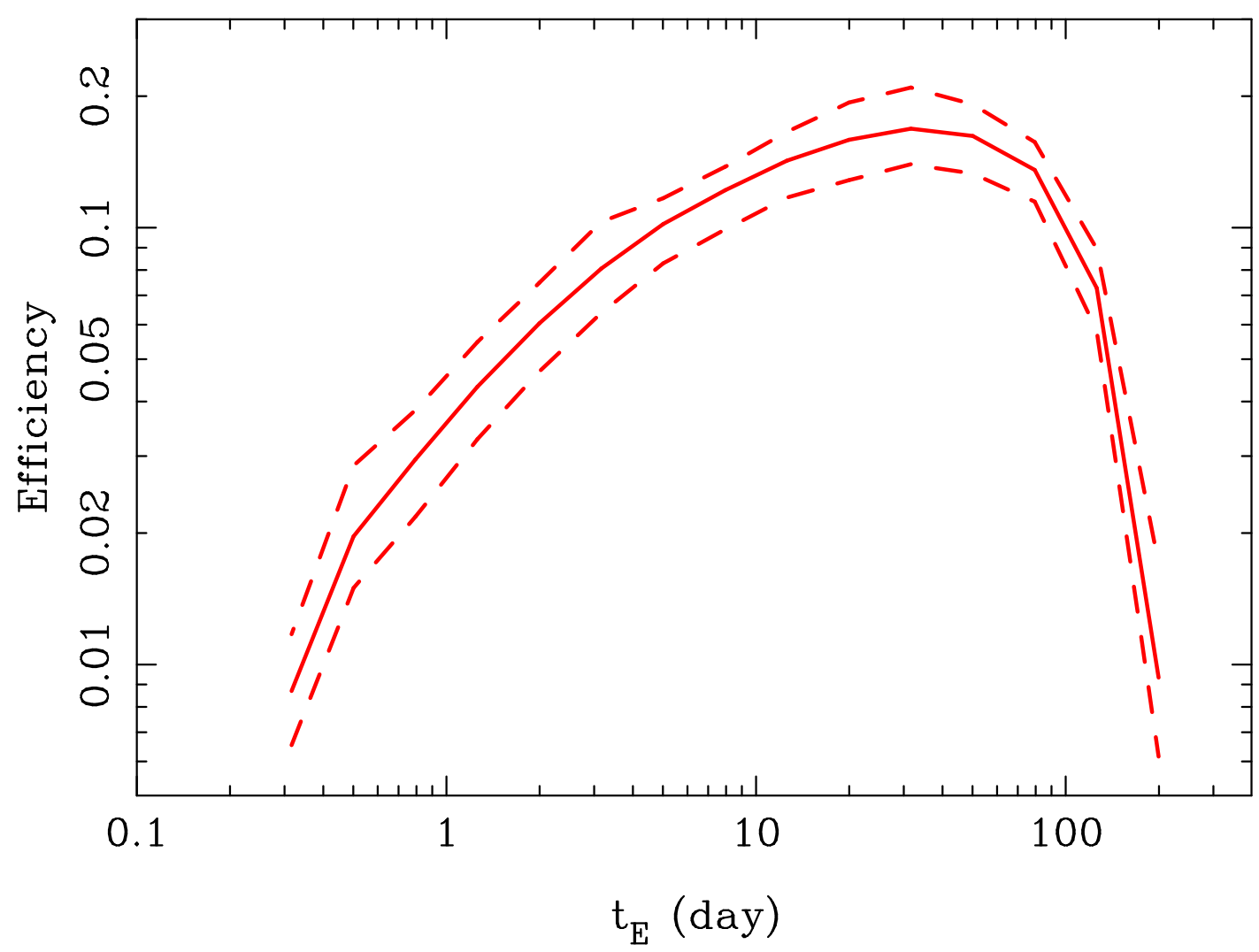

Figure 2.- The detection efficiencies of our experiment as a function of $t_{\mathrm{E}}$ for the source stars down to $I=20.0$ mag. Solid and dashed lines indicate the mean, minimum and maximum efficiencies of all fields. 


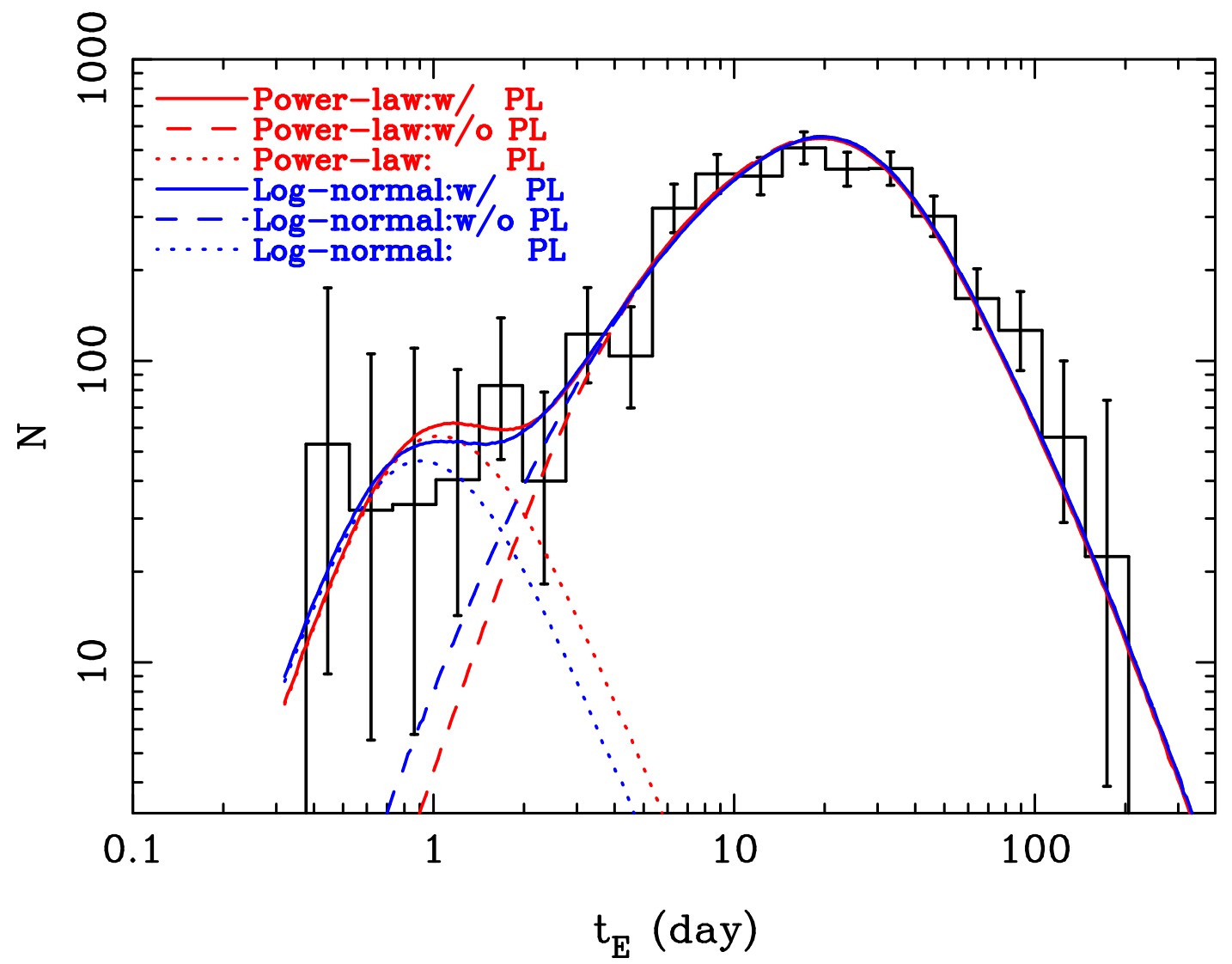

Figure 3.- Observed and theoretical distributions of the event timescale $t_{\mathrm{E}}$. The black histogram represents the observed 474 microlensing events. This is the same data that is shown in Fig. 2 of the main paper, but here it is the data and not the models that are corrected for detection efficiencies. The error bars indicate s.e.m.. The best-fit models with the (1) Power-law and (2) Log-normal mass functions are indicated in red and blue, respectively. For each model, dashed lines indicate models for stellar, stellar remnant and brown dwarf populations and dotted lines represent the Planetary-mass population (PL). Solid lines are the sum of these populations. 


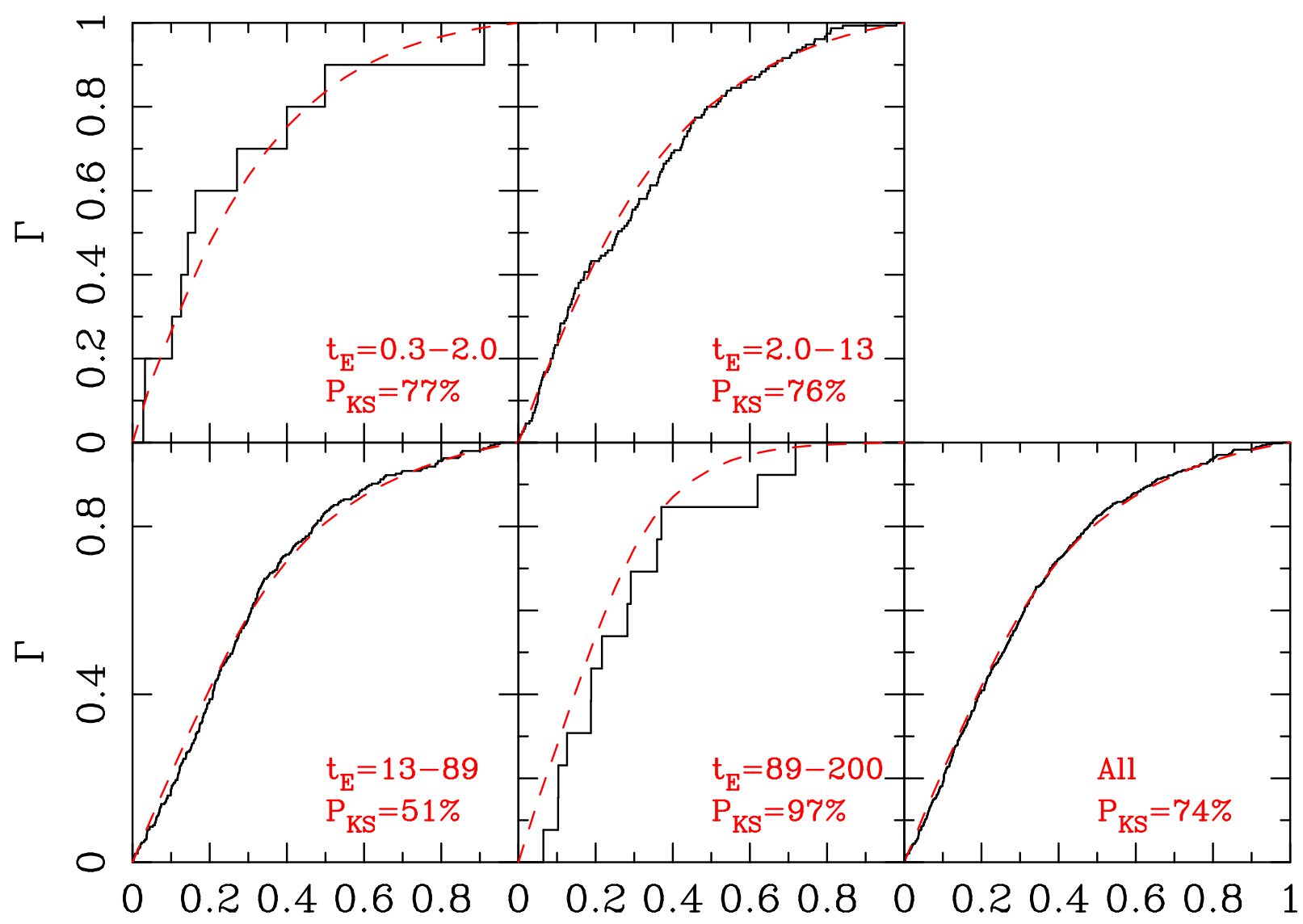

$\mathrm{u}_{0} \quad \mathrm{u}_{0} \quad \mathrm{u}_{0}$

Figure 4.- Cumulative distribution of the impact parameters, $u_{0}$, for the observed sample of 474 microlensing events (histograms) split into different $t_{\mathrm{E}}$ bins. Dashed red curves indicate the expected distribution from the simulation in each $t_{\mathrm{E}}$. The probability that the observed samples are compatible with the model are estimated with the Kolmogorov-Smirnov test, which yields probabilities, $P_{\mathrm{KS}}$, of $77 \%, 76 \%, 51 \%, 97 \%$ and $74 \%$ for the $t_{\mathrm{E}}$ ranges $t_{\mathrm{E}}=0.3-$ $2.0,2.0-13,13-69,89-200$ days and all sample, respectively. The detection efficiency is lower for events with large $u_{0}$ (low magnification). For the longest $t_{\mathrm{E}}$ bin, we expect relatively large $\mathrm{S} / \mathrm{N}$ even for large $u_{0}$. However, we have set a window of 120 -days in cut-1, which the estimates the $\mathrm{S} / \mathrm{N}$ by comparing to the deviation outside of the window. For the long $t_{\mathrm{E}}$, the tails of events extend outside of the window and decrease the $\mathrm{S} / \mathrm{N}$. This decreases the detection efficiency for events large $u_{0}$ and $t_{\mathrm{E}}$. 


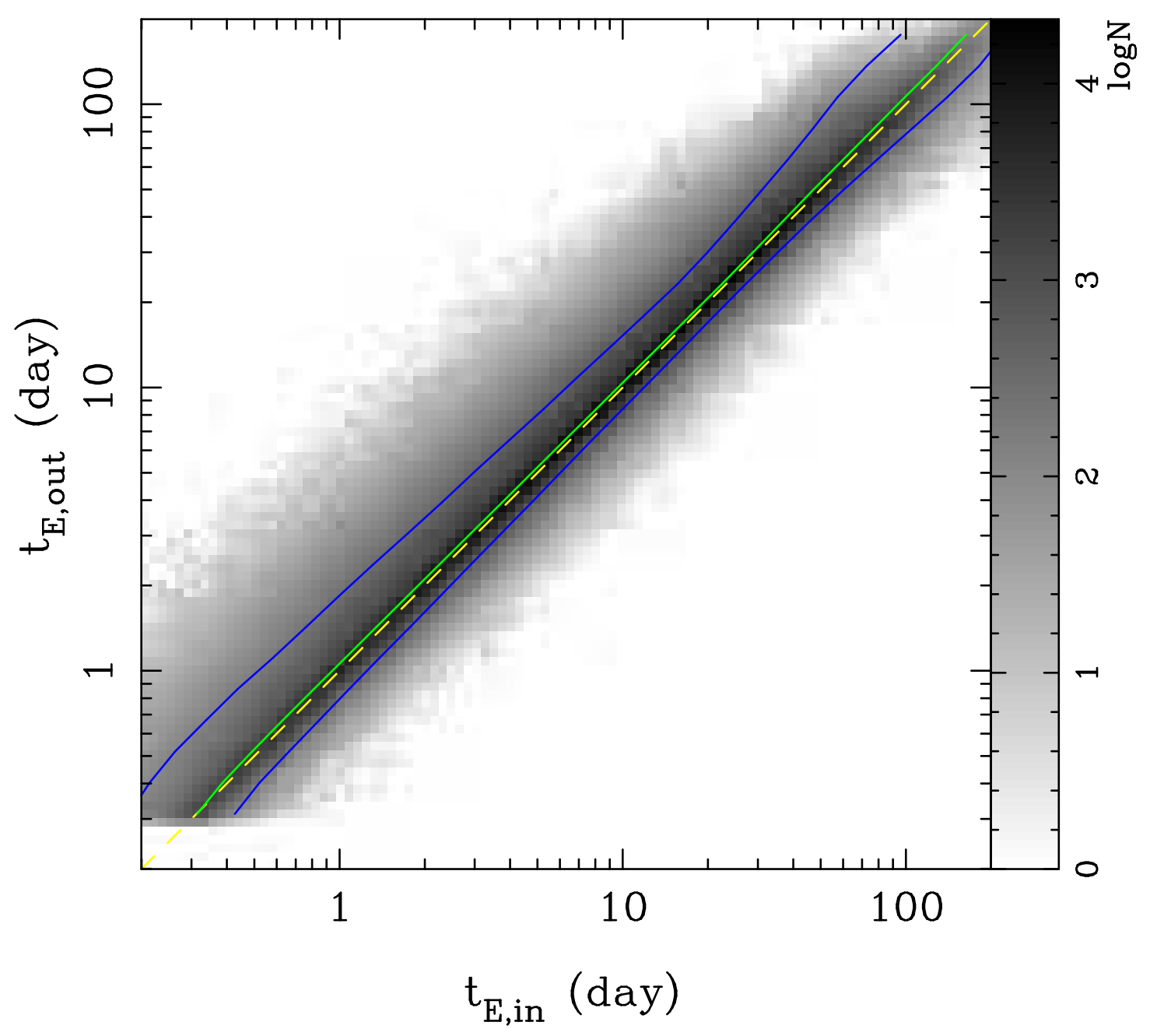

Figure 5.- The output timescale, $t_{\mathrm{E}, \text { out }}$, as a function of input $t_{\mathrm{E}, \text { in }}$ of artificial events in our event simulations. The number density of events in each bin is shown as a logarithmic grayscale, as indicated on the right. The artificial evens were generated uniformly in magnitude and $\log \left(t_{\mathrm{E}}\right)$. Only events which passed the selection criteria are presented, and we have not weighted by the Luminosity function or true $t_{\mathrm{E} \text {,in }}$ distribution. The yellow dashed line indicates $t_{\mathrm{E} \text {,in }}=t_{\mathrm{E} \text {,out }}$, and the green solid line represents the mean of $t_{\mathrm{E} \text {,in }}$ as a function of a mean of $t_{\mathrm{E} \text {,out }}$. We found $t_{\mathrm{E} \text {,in }}$ is systematically $\sim 5 \%$ smaller than $t_{\mathrm{E} \text {,out }}$ regardless of $t_{\mathrm{E}}$. The blue solid lines indicate $90 \%$ interval in each $t_{\mathrm{E} \text {,out }}$ bin. 


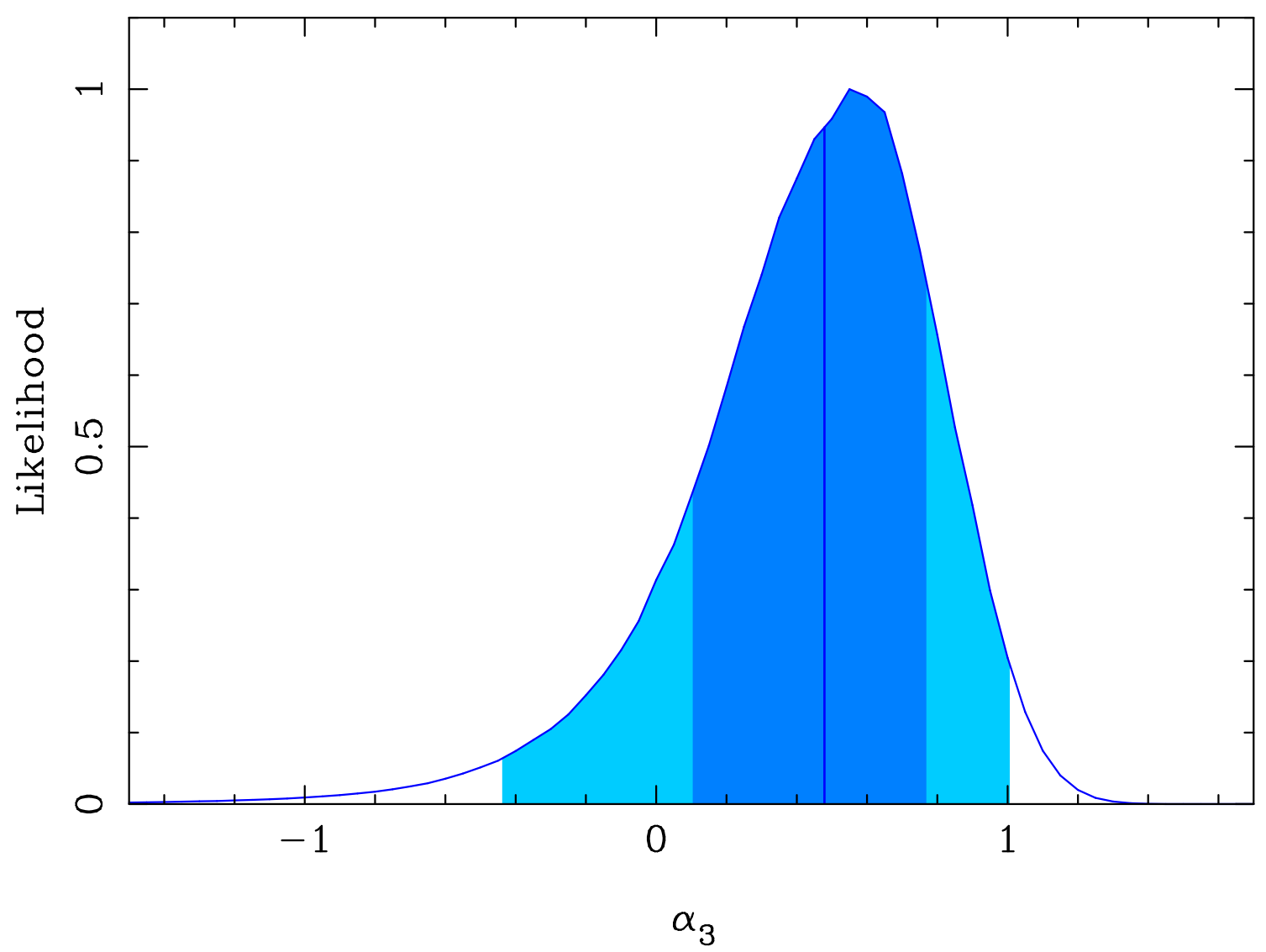

Figure 6.- Likelihood distribution for the Power-law index $\alpha_{3}$ in brown dwarf regime without the planetary mass population, including only the events with $t_{\mathrm{E}}>2$ days. The vertical lines and colored regions indicate the median $\left(\alpha_{3}=0.48\right)$ and $68 \%\left(\alpha_{3}=0.10\right.$ and $0.77)$ and $95 \%\left(\alpha_{3}=-0.44\right.$ and 1.01) confidence intervals. 


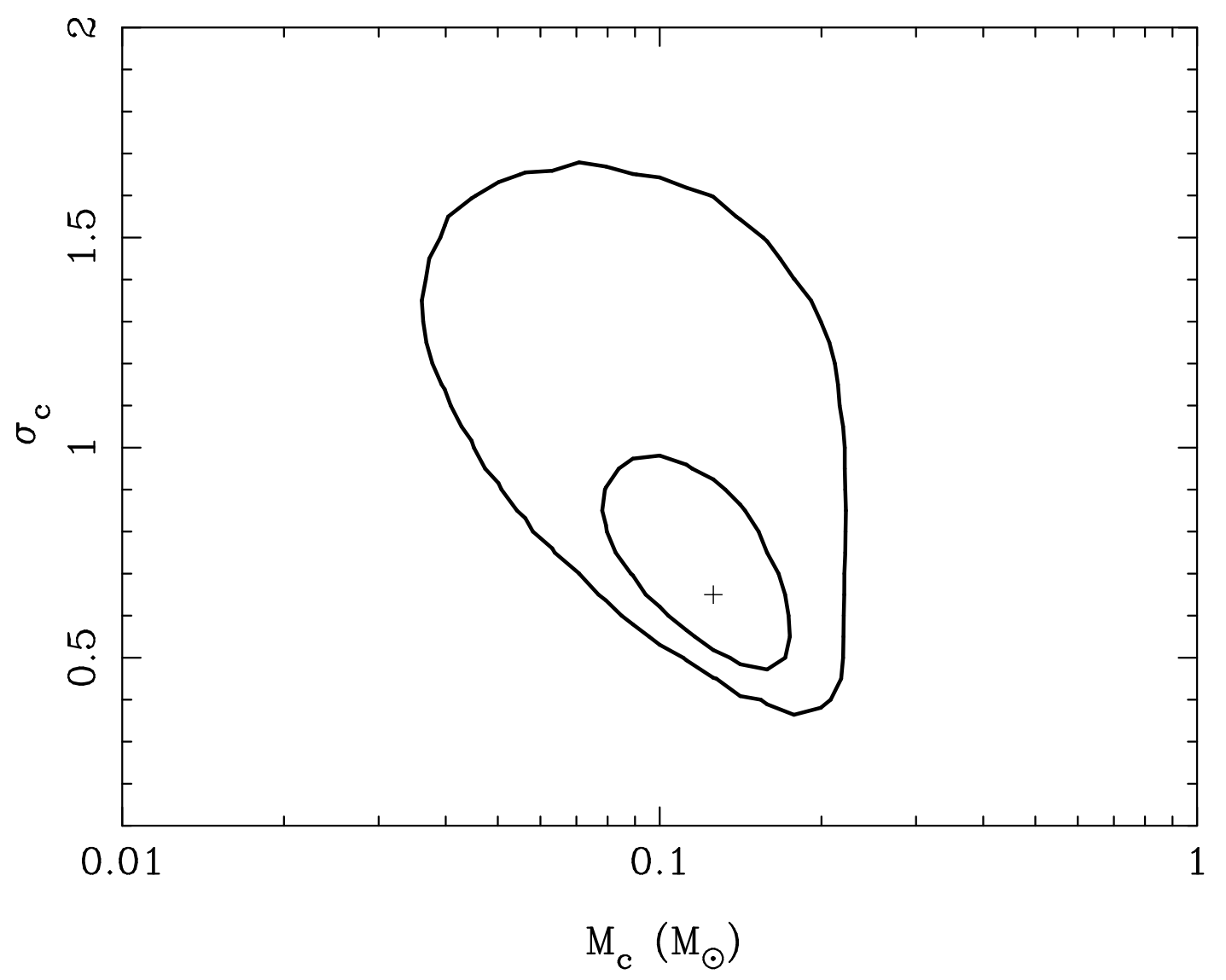

Figure 7.- Likelihood contours for the mean mass, $M_{\mathrm{c}}$, and variance, $\sigma_{\mathrm{c}}$ of log-normal mass functions without the planetary mass population, including only the events with $t_{\mathrm{E}}>2$ days. Two contours indicate the $68 \%$ and $95 \%$ confidence intervals. "+" indicate the maximum likelihood point. The median and $68 \%$ confidence intervals are $M_{\mathrm{c}}=0.12_{-0.03}^{+0.03}$ and $\sigma_{\mathrm{c}}=$ $0.76_{-0.16}^{+0.27}$ and these are consistent with previous work ${ }^{22}$. 


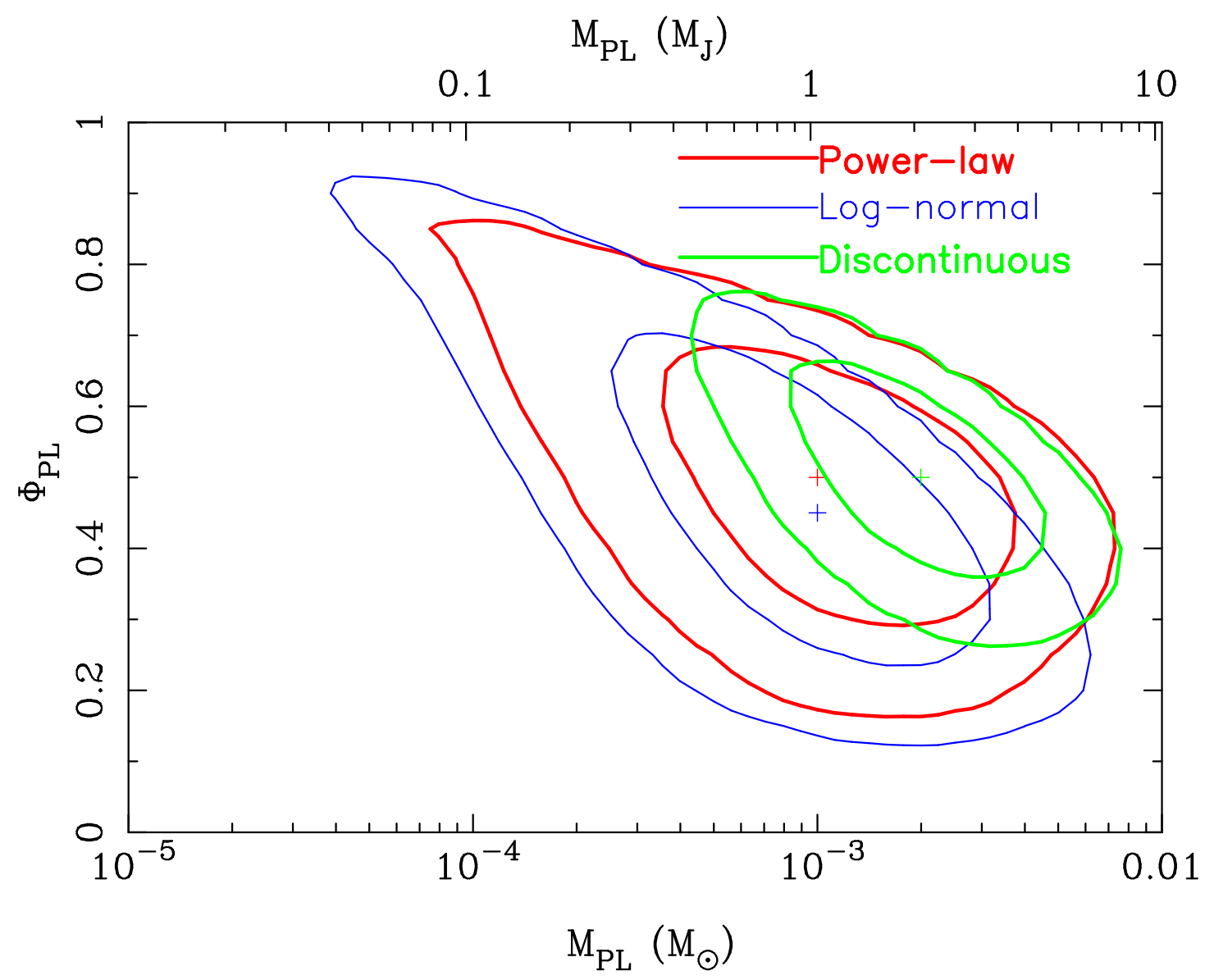

Figure 8.- Likelihood contours for the planetary mass function parameters: the fraction of all objects that are in the planetary mass population, $\Phi_{\mathrm{PL}}$, and their mass, $M_{\mathrm{PL}}$, assuming a $\delta$-function mass function. The two contours in each color indicate the $68 \%$ and $95 \%$ confidence levels. "+" indicate the maximum likelihood points. The red and blue contours represent the Power-law (1) and log-normal (2) mass functions also shown Fig. 3 of the main paper. The green lines indicate the discontinuous mass function model $(3)^{\underline{24}, 61}$, which uses the universal stellar mass function and discontinuous brown dwarf mass function with a power-law index of $\alpha_{3}=0.3$ and a scale of $R_{\mathrm{HBL}}=0.3$. The planetary mass functions are consistent each other despite the different brown dwarf mass functions. 


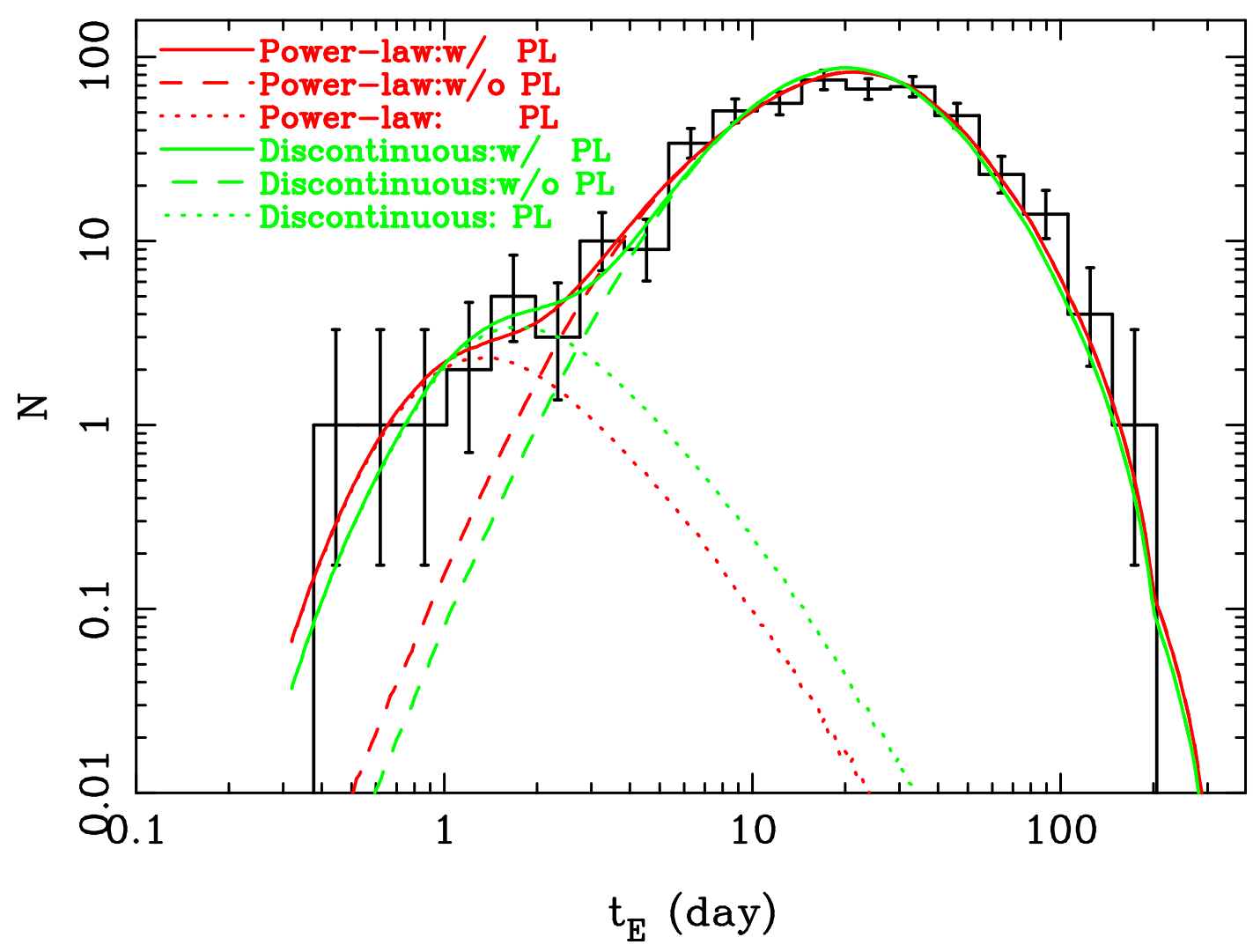

Figure 9.- Observed and theoretical distributions in the timescale $t_{\mathrm{E}}$ as in Fig. 2 of the main paper. The black histogram represents the observed 474 microlensing events with the error bars indicating s.e.m.. The green lines indicates the best-fit for the discontinuous brown dwarf mass function $\underline{24}, \underline{61}$ model (3) with $R_{\mathrm{HBL}}=0.3$. The red line indicates the model (1) Power-law mass function. In each model, dashed lines indicate models for stellar, stellar remnant and brown dwarf populations. Dotted lines represent the Planetary-mass population (PL). Solid lines are the sum of these populations. Although the discontinuous brown dwarf model is a poorer fit to the data than model (1), the fit on the low-mass end is reasonable, and the mass function for the planetary mass objects is consistent with the results for models (1) and (2), which are presented in the main paper. 


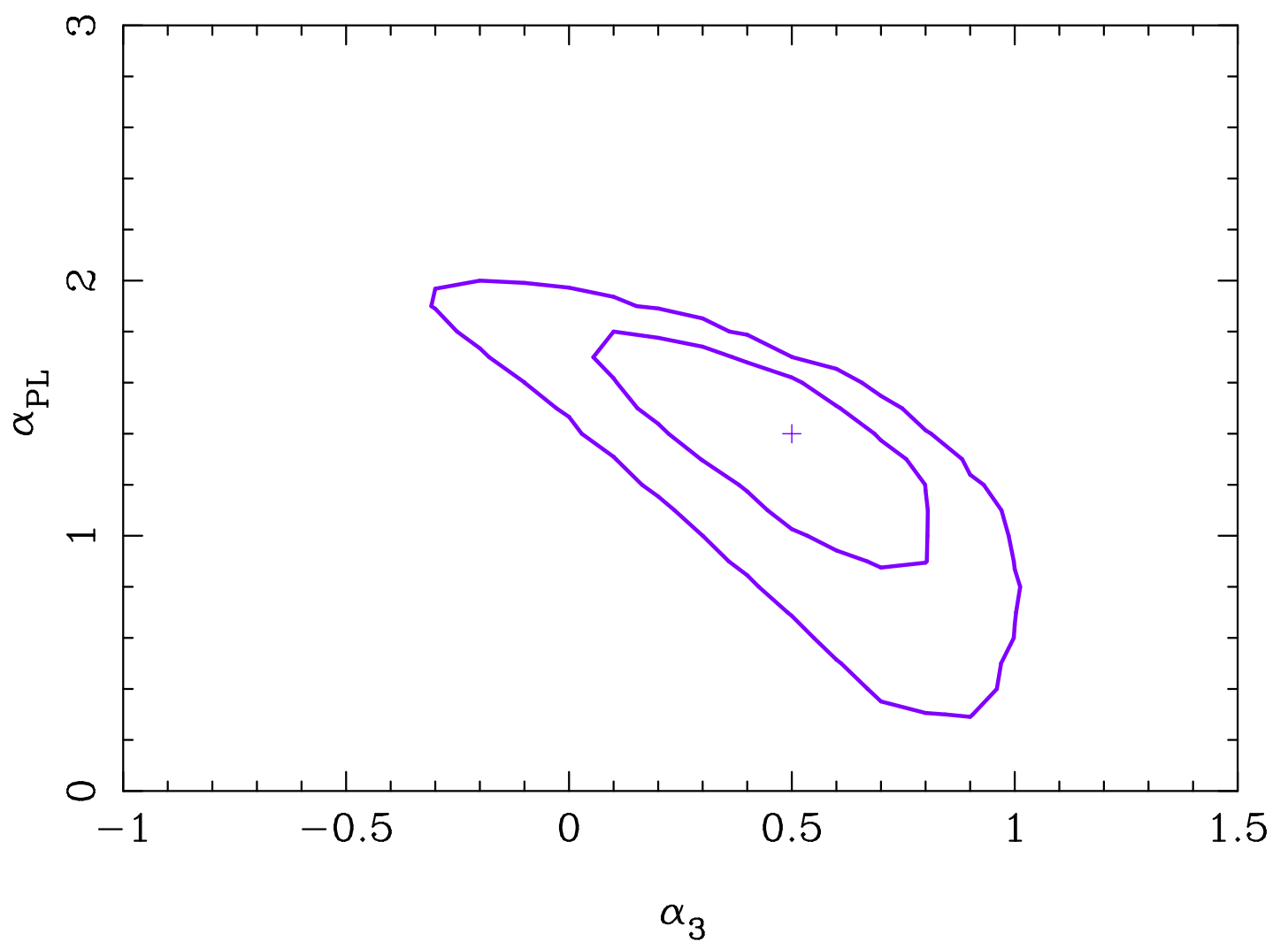

Figure 10.- Likelihood contours for the Power-law indices $\alpha_{3}$ in the brown dwarf regime, and $\alpha_{\mathrm{PL}}$ in the planetary-mass regime. These brown dwarf and planetary mas regimes span the mass ranges $0.01 \leq M / M_{\odot} \leq 0.08$ and $10^{-5}<M / M_{\odot}<0.01$, respectively. The two contours indicate the $68 \%$ and $95 \%$ confidence levels, and "+" indicate the maximum likelihood point. These results do not depend on the lower mass limit of $10^{-5} M_{\odot}$ as the sensitivity to masses lower than $10^{-4} M_{\odot}$ is poor due to the small detection efficiencies at low $t_{\mathrm{E}}$. The $\alpha_{3}$ distribution is consistent with the $\alpha_{3}$ distribution for model (1) with and without the $\delta$-function planetary mass function (see Table 3). The value $\alpha_{\mathrm{PL}}$ is much steeper than $\alpha_{3}$, indicating that this planetary mass objects are separate population from brown dwarfs. 


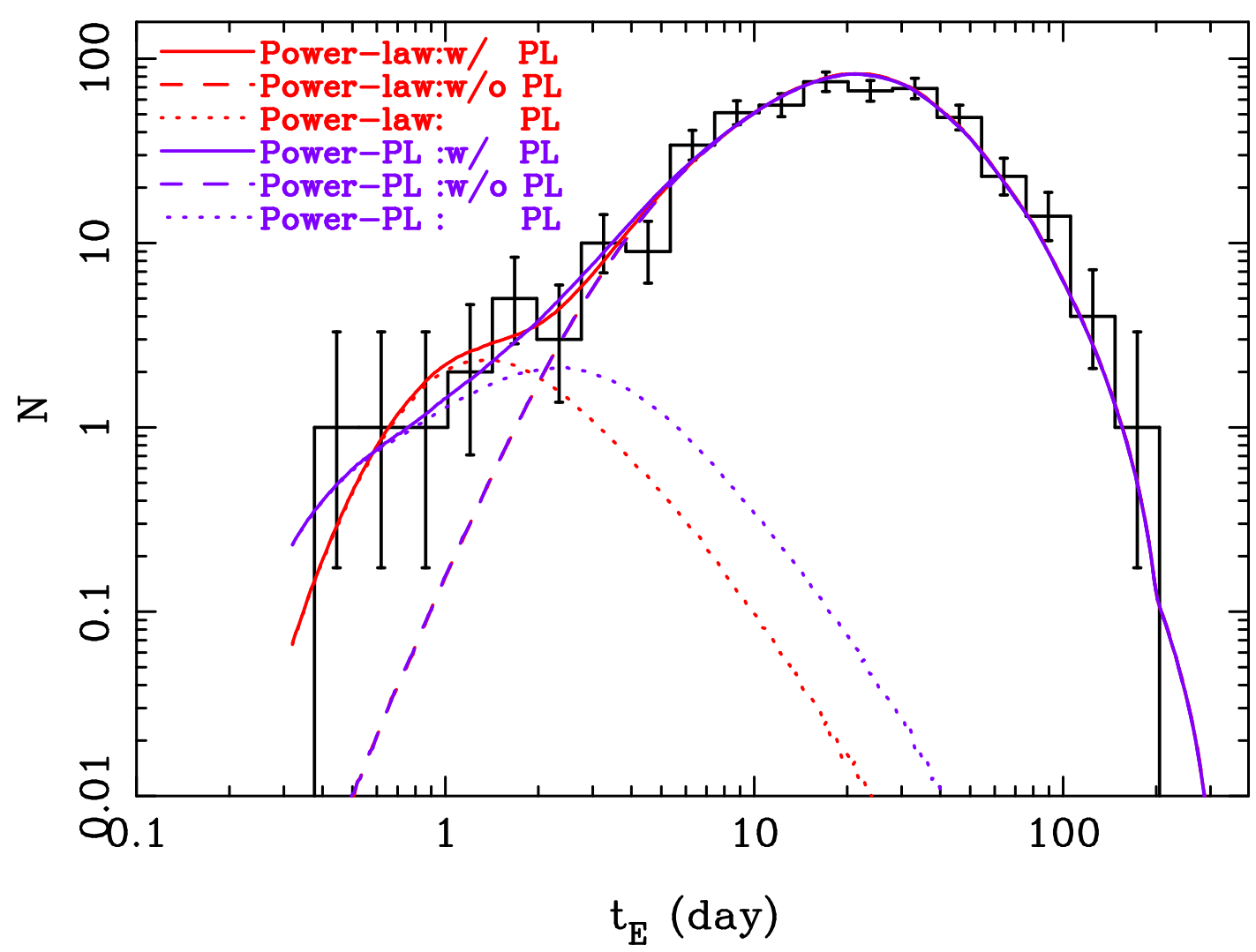

Figure 11.- Observed and theoretical distributions of the timescale $t_{\mathrm{E}}$. The black histogram represents the observed 474 microlensing events with the error bars indicating s.e.m.. The purple line indicates the best-fit model (4) with a power-law stellar and substellar mass function, as in model (1), but with a continuous power-law mass function for in the planetary mass regime, $10^{-5}<M / M_{\odot} \leq 0.08$ (Power-PL), instead of $\delta$-function mass function of model (1). The red line indicates the power-law mass function model (1) with a $\delta$-function planetary mass function. For each model, dashed lines indicate models for stellar, stellar remnant and brown dwarf populations, and dotted lines represent the Planetary-mass population. Solid lines are the sum of these populations. This power-law planetary mass function model (3) has a slightly smaller likelihood value than model (1), but because it has one fewer parameter, it is slightly favored, formally. 
Table 1. MOA-II Galactic bulge fields

\begin{tabular}{llll}
\hline \hline Field & R.A.(2000) & Dec.(2000) & $N_{\text {frame }}$ \\
& & & \\
\hline gb1 & $17: 47: 31.41$ & $-34: 14: 31.09$ & 2,253 \\
gb2 & $17: 54: 01.41$ & $-34: 29: 31.09$ & 2,386 \\
gb3 & $17: 54: 01.41$ & $-32: 44: 31.09$ & 2,067 \\
gb4 & $17: 54: 01.41$ & $-30: 59: 31.09$ & 2,985 \\
gb5 & $17: 54: 01.41$ & $-29: 14: 31.09$ & 8,229 \\
gb6 & $17: 54: 01.41$ & $-27: 29: 31.09$ & 1,779 \\
gb7 & $18: 00: 01.41$ & $-32: 44: 31.09$ & 1,970 \\
gb8 & $18: 00: 01.41$ & $-30: 59: 31.09$ & 2,139 \\
gb9 & $18: 00: 01.41$ & $-29: 14: 31.09$ & 8,301 \\
gb10 & $18: 00: 01.41$ & $-27: 29: 31.09$ & 1,992 \\
gb11 & $18: 06: 01.41$ & $-32: 44: 31.09$ & 2,004 \\
gb12 & $18: 06: 01.41$ & $-30: 59: 31.09$ & 1,790 \\
gb13 & $18: 06: 01.41$ & $-29: 14: 31.09$ & 1,811 \\
gb14 & $18: 06: 01.41$ & $-27: 29: 31.09$ & 1,770 \\
gb15 & $18: 06: 01.41$ & $-25: 44: 31.09$ & 1,952 \\
gb16 & $18: 12: 01.41$ & $-29: 14: 31.09$ & 1,756 \\
gb17 & $18: 12: 01.41$ & $-27: 29: 31.09$ & 1,792 \\
gb18 & $18: 12: 01.41$ & $-25: 44: 31.09$ & 1,799 \\
gb19 & $18: 18: 01.41$ & $-25: 29: 31.09$ & 1,704 \\
gb20 & $18: 18: 01.41$ & $-23: 44: 31.09$ & 1,679 \\
gb21 & $18: 18: 01.41$ & $-21: 59: 31.09$ & 1,659 \\
gb22 & $18: 36: 25.41$ & $-23: 53: 31.09$ & 1,782 \\
\hline & & & \\
\hline
\end{tabular}

Note. - $N_{\text {frame }}$ indicates the number of observed frames (exposures) during 2006-2007, which are used in this analysis. Fields gb5 and gb9 were observed with a 10 minute cadence, while others were observed with a 50 minute cadence. 
Table 2. Event Selection Criteria

\begin{tabular}{lll}
\hline \hline level & \multicolumn{1}{c}{ criteria } & \\
& & \\
\hline cut0 & $N_{\text {detect }} \geq 3$ & Number of frames in which the object is detected. \\
\hline cut1 & $N_{\text {data }} \geq 500$ & Number of data points \\
& $N_{\text {out }} \geq 10$ & Number of data points outside of the 120-day window \\
& $\chi_{\text {out }}^{2} /$ dof $\leq 3$ & $\chi^{2}$ outside of the 120 -day window \\
& $N_{\text {bump }} \geq 1$ & Number of bumps in the window, where a bump \\
& $\chi_{3+}=\Sigma_{i}\left(F_{i}-F_{\text {base }}\right) / \sigma_{i}^{\prime} \geq 80$ & has $>3$ consecutive points $>3 \sigma^{\prime}$ above baseline \\
& Total significance of consecutive points with $>3 \sigma^{\prime}$ \\
\hline cutt 2 & Fits never converge if parameters are degenerate \\
& $\chi^{2} /$ dof $\leq 2$ & $\chi^{2}$ for all data \\
& $\chi_{1}^{2} /$ dof $\leq 2$ & $\chi^{2}$ for $|t| \leq t_{\mathrm{E}}$ \\
& $\chi_{2}^{2} /$ dof $\leq 2$ & $\chi^{2}$ for $|t| \leq 2 t_{\mathrm{E}}$ \\
$0.3 \leq t_{\mathrm{E}} \leq 200$ days & Einstein radius crossing timescale \\
$\sigma_{t_{\mathrm{E}}} / t_{\mathrm{E}} \leq 0.5$ & Error in $t_{\mathrm{E}}$ \\
$\sigma_{t_{\mathrm{E}}} \leq 12$ days & Error in $t_{\mathrm{E}}$ \\
$3824 \leq t_{0} \leq 4420 \mathrm{JD}$ & Peak should be within observational period \\
$u_{0} \leq 1$ & The minimum impact parameter \\
$\sigma_{u_{0}} \leq 0.3$ & Error in $u_{0}$ \\
$I_{s} \leq 20.0$ & Apparent $I$-band source magnitude \\
$\left(F_{\mathrm{s}}-F_{\text {cat }}\right) / F_{\text {cat }} \leq 3$ & Source flux should not greatly exceed catalog flux \\
$\chi_{3+} \geq 70 N_{2 \sigma}-500$ & Exclude systematic residuals (depending on \\
& total significance) \\
$\chi_{3+} \geq 45 N_{3 \sigma}$ OR $N_{3 \sigma} \leq 2$ & same as above \\
\hline
\end{tabular}

Note. - JD'=JD-2450000. $\sigma_{i}^{\prime} \equiv \sigma_{i} \sqrt{\chi_{\text {out }}^{2} / \text { dof. }} . N_{2 \sigma}$ and $N_{3 \sigma}$ represent the maximum number of consecutive measurements which are scattered from the best fit model with an excess flux of more than 2- $\sigma$ and $3-\sigma$, respectively. 
Table 3. Mass Function

\begin{tabular}{|c|c|c|c|c|}
\hline \# & $\begin{array}{l}\text { Mass } \\
\left(M_{\odot}\right)\end{array}$ & Function & $\begin{array}{c}\text { parameter } \\
\left(M \text { and } \sigma \text { are in } M_{\odot}\right)\end{array}$ & $\begin{array}{l}\text { Fraction } \\
\left(N_{*}\right)\end{array}$ \\
\hline \multirow[t]{8}{*}{1} & $40.0 \leq M$ & Gaussian & Black hole $\left(M_{\mathrm{r}}=5, \sigma_{\mathrm{r}}=1\right)$ & 0.0031 \\
\hline & $8.00 \leq M \leq 40.0$ & Gaussian & Neutron $\operatorname{star}\left(M_{\mathrm{r}}=1.35, \sigma_{\mathrm{r}}=0.04\right)$ & 0.021 \\
\hline & $1.00 \leq M \leq 8.00$ & Gaussian & White dwarf $\left(M_{\mathrm{r}}=0.6, \sigma_{\mathrm{r}}=0.16\right)$ & 0.18 \\
\hline & $0.70 \leq M \leq 1.00$ & Power-law & $\alpha_{1}=2.0$ & 1.0 \\
\hline & $0.08 \leq M \leq 0.70$ & Power-law & $\alpha_{2}=1.3$ & \\
\hline & $0.01 \leq M \leq 0.08$ & Power-law* & $\alpha_{3}=0.48_{-0.37}^{+0.29} \mathrm{w} / \mathrm{o} \mathrm{PL}$ & $0.73_{-0.19}^{+0.22}$ \\
\hline & $0.01 \leq M \leq 0.08$ & Power-law** & $\alpha_{3}=0.50_{-0.60}^{+0.36} \mathrm{w} / \mathrm{PL}$ & $0.74_{-0.27}^{+0.30}$ \\
\hline & $M=M_{\mathrm{PL}}$ & $\delta$-function ${ }^{* *}$ & $M_{\mathrm{PL}}=1.1_{-0.6}^{+1.2} \times 10^{-3}, \Phi_{\mathrm{PL}}=0.49_{-0.13}^{+0.13}$ & $1.9_{-0.8}^{+1.3}$ \\
\hline \multirow[t]{7}{*}{2} & $40.0 \leq M$ & Gaussian & Black hole $\left(M_{\mathrm{r}}=5, \sigma_{\mathrm{r}}=1\right)$ & 0.0031 \\
\hline & $8.00 \leq M \leq 40.0$ & Gaussian & Neutron star $\left(M_{\mathrm{r}}=1.35, \sigma_{\mathrm{r}}=0.04\right)$ & 0.021 \\
\hline & $1.00 \leq M \leq 8.00$ & Gaussian & White dwarf $\left(M_{\mathrm{r}}=0.6, \sigma_{\mathrm{r}}=0.16\right)$ & 0.18 \\
\hline & $0.08 \leq M \leq 1.00$ & Log-normal ${ }^{*}$ & $M_{\mathrm{c}}=0.12_{-0.03}^{+0.03}, \sigma_{\mathrm{c}}=0.76_{-0.16}^{+0.27}$ & 1.0 \\
\hline & $0.01 \leq M \leq 0.08$ & Log-normal* ${ }^{*}$ & $M_{\mathrm{c}}=0.12_{-0.03}^{+0.03}, \sigma_{\mathrm{c}}=0.76_{-0.16}^{+0.27}$ & $0.70_{-0.30}^{+0.19}$ \\
\hline & $0.00 \leq M \leq 0.01$ & Log-normal ${ }^{*}$ & $M_{\mathrm{c}}=0.12_{-0.03}^{+0.03}, \sigma_{\mathrm{c}}=0.76_{-0.16}^{+0.27}$ & $0.17_{-0.15}^{+0.24}$ \\
\hline & $M=M_{\mathrm{PL}}$ & $\delta$-function $* * *$ & $M_{\mathrm{PL}}=0.83_{-051}^{+0.96} \times 10^{-3}, \Phi_{\mathrm{PL}}=0.46_{-015}^{+0.17}$ & $1.8_{-08}^{+1.7}$ \\
\hline \multirow[t]{7}{*}{3} & $40.0 \leq M$ & Gaussian & Black hole $\left(M_{\mathrm{r}}=5, \sigma_{\mathrm{r}}=1\right)$ & 0.00060 \\
\hline & $8.00 \leq M \leq 40.0$ & Gaussian & Neutron star $\left(M_{\mathrm{r}}=1.35, \sigma_{\mathrm{r}}=0.04\right)$ & 0.0061 \\
\hline & $1.00 \leq M \leq 8.00$ & Gaussian & White dwarf $\left(M_{\mathrm{r}}=0.6, \sigma_{\mathrm{r}}=0.16\right)$ & 0.097 \\
\hline & $0.50 \leq M \leq 1.00$ & Power-law & $\alpha_{1}=2.3$ & 1.0 \\
\hline & $0.075 \leq M \leq 0.50$ & Power-law & $\alpha_{2}=1.3$ & \\
\hline & $0.01 \leq M \leq 0.075$ & Power-law & $\alpha_{3}=0.3, R_{\mathrm{HBL}}=0.3$ & 0.19 \\
\hline & $M=M_{\mathrm{PL}}$ & $\delta$-function & $M_{\mathrm{PL}}=1.9_{-0.9}^{+1.4} \times 10^{-3}, \Phi_{\mathrm{PL}}=0.50_{-0.10}^{+0.11}$ & $1.3_{-0.4}^{+0.7}$ \\
\hline \multirow[t]{3}{*}{4} & $0.08 \leq M$ & & same as model (1) & \\
\hline & $0.01 \leq M \leq 0.08$ & Power-law** & $\alpha_{3}=0.49_{-0.27}^{+0.24} \mathrm{w} / \mathrm{PL}$ & $0.73_{-0.15}^{+0.17}$ \\
\hline & $10^{-5} \leq M \leq 0.01$ & Power-law** & $\alpha_{\mathrm{PL}}=1.3_{-0.4}^{+0.3} \mathrm{w} / \mathrm{PL}$ & $5.5_{-4.3}^{+18.1}$ \\
\hline
\end{tabular}

Note. - \# is the model ID number: (1) power-law $+\delta$-function, (2) log-normal $+\delta$-function, (3) discontinuous power-law $+\delta$-function and (4) power-law+power-law. Gaussian: $d N / d M=\exp \left[\left(M-M_{\mathrm{r}}\right)^{2} /\left(2 \sigma_{\mathrm{r}}^{2}\right)\right]$. Function types are: Power-law: $d N / d \log M=M^{1-\alpha}$. Log-normal: $d N / d \log M=\exp \left[\left(\log M-\log M_{\mathrm{c}}\right)^{2} /\left(2 \sigma_{\mathrm{c}}^{2}\right)\right]$. Fraction is the number of objects relative to the number of main sequence stars with $0.08 \leq M / M_{\odot} \leq 1, N_{*}$ $\left(0.075 \leq M / M_{\odot} \leq 1\right.$ for the model 3$)$. The number of stellar remnants is estimated by extending the upper main sequence Power-law $\alpha=2.0(\alpha=2.3$ for the model 3$)$ through this higher mass regime. $*: \alpha_{3}$ or $\left(M_{\mathrm{PL}}\right.$, $\left.\Phi_{\mathrm{PL}}\right)$ are fit to events with $t_{\mathrm{E}}>2$ days without the planetary mass $\delta$-function. $* *: \alpha_{3}$ and $\left(M_{\mathrm{PL}}, \Phi_{\mathrm{PL}}\right)\left(\alpha_{\mathrm{PL}}\right.$ for the model 3) are fit simultaneously for the full sample. $* * *: M_{\mathrm{c}}=0.12$ and $\sigma_{\mathrm{c}}=0.76$ are held fixed when fitting $M_{\mathrm{PL}}$ and $\Phi_{\mathrm{PL}}$. The fraction of planetary mass objects in model (4) is large because it extends down to $M=10^{-5} M_{\odot}$ where have no sensitivity. 